\title{
Simulación y engaño académico: En Respuesta a "RéPlica a Julio A. Piña LÓPEZ [...]" DE JOSÉ CONCEPCIÓN GAXIOLA ROMERO
}

\author{
Julio Alfonso Piña López \\ Investigador independiente \\ México
}

\begin{abstract}
RESUMEN
En 2017 se publicó un artículo de nuestra autoría, en el que revisamos exhaustivamente tres trabajos de investigación publicados por José Concepción Gaxiola Romero y colaboradores entre 2012 y 2013. Los hallazgos principales que se reportaron fueron en esencia dos: por un lado, la repetición de más de una veintena de párrafos -iguales o con ligeras modificaciones-en los tres trabajos; por el otro, la fabricación y/o falsificación deliberada de los datos en dos de los tres. Recientemente apareció una supuesta réplica de Gaxiola, de cuya lectura se esperaba una basada en una sólida argumentación y en la presentación de evidencia tangible de que nuestros hallazgos estaban equivocados o que eventualmente se había malinterpretado algo; antes bien, aquél desvió la atención hacia otros asuntos que no tuvieron nada que ver con lo publicado por nosotros en 2017. En esta respuesta a su supuesta réplica, nos damos a la tarea de presentar evidencia adicional de cómo Gaxiola y colaboradores practican frecuentemente conductas éticamente inaceptables y conductas éticamente cuestionables, haciendo de la simulación y el engaño académico un modo de vida.
\end{abstract}

Palabras Clave:

Conductas Éticamente Inaceptables, Conductas Éticamente Cuestionables, Simulación, Engaño Académico

\section{SIMULATION AND ACADEMIC DECEPTION: IN RESPONSE TO "REPLY TO JULIO A. PIÑa LóPez [...]” BY José CONCEPCIÓN GAXIOLA Romero}

\begin{abstract}
In 2017, an article of our authorship was published in, which consisted of an exhaustive review of three research works published by José Concepción Gaxiola Romero and collaborators between 2012 and 2013. The main findings that we reported were two in essence: on the one hand, the repetition of more than twenty paragraphs -equal or with slight modifications-in the three papers; on the other, the manufacture and/or falsification of data in two of the three. Recently, a supposed reply of Gaxiola was published, from whose reading we expected one based on a solid argumentation and the presentation of tangible evidence that our findings were wrong or that had eventually misunderstood something; notwithstanding, he diverted the attention on other matters that had nothing to see with the findings published by us in 2017 . In this answer to the supposed reply, we will present additional evidence of how Gaxiola and collaborators are practicing ethically unacceptable behaviors and ethically questionable behaviors, doing of both simulation and academic deception a way of life.
\end{abstract}

Keywords:

Ethically Unacceptable Behavior, Ethically Questionable Behavior, Simulation, Academic Deception

Bitácora del Artículo:

| Recibido: 1 de Febrero de 2019 | Aceptado: 1 Abril de 2019 | Publicado en línea: Enero-Junio de 2019 | 


\section{Simulación y engaño aCAdémico: En Respuesta a "RÉPlica a Julio A. Piña LÓPEZ [...]" DE JOSÉ CONCEPCIÓN GAXIOLA ROMERO

\author{
Julio Alfonso Piña López \\ Investigador independiente \\ México
}

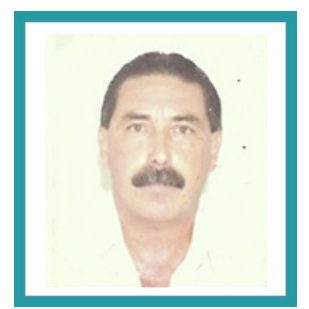

Julio Alfonso Piña-López

Investigador independiente

Correo: pina.lopez.julio@gmail.com

Licenciado en Psicología, con estudios de Maestría en Psicología por la Universidad de Sonora. Ha sido Profesor-Investigador en el Departamento de Psicología y Ciencias de la Comunicación y ha participado en la misma institución como Jefe Administrativo del Programa de Salud Institucional.

\section{CONTRIBUCIÓN DEL AUTOR}

Se atribuye al autor la responsabilidad única sobre el trabajar al concibir y desarrollar los puntos de vista que en este trabajo se presentan.

\section{DATOS DE Filiación del AUtOR}

Investigador independientes

\section{(c)

Este es un artículo de acceso abierto distribuido bajo los términos de la licencia Creative Commons Reconocimiento-NoComercial 4.0 Internacional, por lo que su contenido gráfico y escrito se puede compartir, copiar y redistribuir total o parcialmente sin necesidad de permiso expreso de su autor con la única condición de que no se puede usar con fines directamente comerciales y los términos legales de cualquier trabajo derivado deben ser los mismos que se expresan en la presente declaración. La única condición es que se cite la fuente con referencia a la Revista Digital Internacional de Psicología y Ciencia Social y a su autor. 


\section{TABLA DE CONTENIDO}

INTRODUCCIÓN

REFERENCIAS

ANEXo

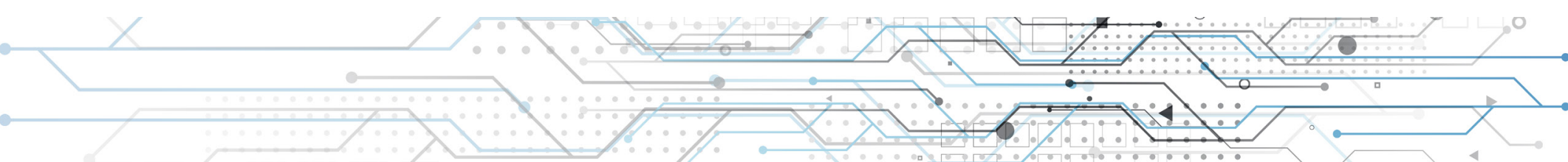




\section{INTRODUCCIÓN}

$\mathrm{E}$ n el Volumen 22, Número 2, de Enseñanza e Investigación en Psicología se publicó un artículo de nuestra autoría, intitulado Predictores del rendimiento académico en Bachilleres, de Gaxiola y colaboradores: ¿Un ejemplo de corrupción académica? (Piña, 2017). Al siguiente año este mereció una supuesta réplica por parte José Concepción Gaxiola Romero en la misma revista (Gaxiola, 2018)' ${ }^{1}$ de cuya lectura esperábamos una que estuviera a la altura de un investigador que pertenece al Sistema Nacional de Investigadores (en lo sucesivo, SNI). Esto es, Gaxiola estaba obligado a presentar una línea de argumentación sólida, demostrando con evidencia tangible en qué y por qué los hallazgos reportados por nosotros estaban equivocados, o si eventualmente habíamos malinterpretado algo. Al dar respuesta a la supuesta réplica de Gaxiola, iremos analizando algunos párrafos en los que este usa de manera sesgada la información; cada uno de ellos lo hemos numerado y varios los hemos resaltado con negritas, con el objeto de poner en evidencia las contradicciones en que incurre aquél. Presentaremos al lector evidencia adicional, haciendo notar también las omisiones deliberadas de Gaxiola -lo que conoce como la No-R_con relación a los dos hallazgos principales que reportamos en 2017.

Los puntos centrales en la supuesta réplica de José Concepción Gaxiola Romero: El arte de morir en el intento... 1. De acuerdo con el Código de Ética del Psicólogo (Sociedad Mexicana de Psicología, 2007), es necesario dar respuesta a los comentarios negativos que se realicen del trabajo personal. En el artículo 142 de dicho código se señala: "Si el psicólogo tiene conocimiento de declaraciones engañosas acerca de su trabajo hechas por otros, hace esfuerzos para corregir tales declaraciones [...] (Gaxiola, 2018; p. 109).

1. Así, la crítica destructiva o las acusaciones directas al trabajo personal no posibilitan la discusión profesional con el ánimo de avanzar en el conocimiento y violan un lineamiento general del ya mencionado Código de Ética del Psicólogo [...] Invitamos a los lectores a revisar las últimas publicaciones de Piña

1 Profesor-Investigador de Tiempo Completo en el Programa Docente de Psicología de la Universidad de Sonora (UNISON), además de ser Profesor con Perfil Deseable PRODEP e Investigador Nacional nivel II.
(Piña, 2015, 2016, 2017), cuya "línea de investigación" y único objetivo de los últimos años ha sido la crítica destructiva de nuestro trabajo de investigación (Gaxiola, 2018; p. 109).

2. Lo que se pretende aportar, más que una respuesta puntual a los infundios de Piña (2017) publicados en esta misma revista [...] (Gaxiola, 2018; p. 110).

En principio, nos sorprende sobremanera el uso no sólo de artículos del Código de Ética del Psicólogo para asumir una defensa de su trabajo, sino también el uso de calificativos personales. Así, dicho sea de paso desconocíamos que argumentar en qué y por qué un autor está equivocado en sus planteamientos teórico-conceptuales, por un lado, así como aportar evidencias sobre los que son consideradas como conductas éticamente inaceptables y éticamente cuestionables (Aluja y Birke, 2004), por el otro, merezca ser evaluado en los términos expuestos por Gaxiola. En efecto, cualquier lector que se tome la molestia de revisar los artículos publicados por Piña en 2015 y 2016, encontrará que el primero de ellos se centró en un análisis del concepto de resiliencia en psicología (Piña, 2015), en tanto que el segundo en el uso y abuso del modelamiento con ecuaciones estructurales en psicología y salud - que derivado de un análisis que hicimos de un trabajo publicado por Gaxiola y colaboradores en 2013 (Piña, 2016). El contenido de ambos artículos fue planteado en términos estrictamente académicos, los cuales, por cierto, nunca tuvieron respuesta en estos mismos términos.

4. En la página 110, y apoyándose en la lectura de especialistas clásicos en metodología, al referirse al subtítulo de nuestro trabajo publicado en 2017, Predictores del rendimiento académico en Bachilleres, de Gaxiola y colaboradores: ¿Un ejemplo de corrupción académica? [...] sostiene que: A partir de lo señalado anteriormente, el objetivo del presente artículo es justificar la viabilidad del uso de una misma base de datos en diferentes productos de investigación. Acto seguido, Gaxiola hace un recuento de cómo en encuestas mundiales ( $p$. 111), estudios derivados de censos (p. 111), encuestas nacionales de salud (p. 111) y estudios longitudinales (pp. 111-112), en todos y cada uno de ellos se menciona el uso de la misma muestra, de la cual derivan varias publicaciones, concluyendo que ello también está permitido en la psicología [...] Lo anterior es especialmente acostumbrado en la investigación multivariada, pero con la condición de que se dé cumplimiento a los diversos 
objetivos específicos de investigación planteados (Gaxiola, 2018; p. 112)2.

El que hayamos cuestionado la lógica teórico-conceptual en la que basó sus trabajos Gaxiola (Piña, 2015, 2016), Ilamando la atención sobre la interpretación errónea que hace de los datos obtenidos (Piña, 2016), o finalmente preguntado si las tres publicaciones que analizamos se constituían en posibles ejemplos de corrupción académica (Piña, 2017), no tienen nada que ver ni con comentarios negativos, ni con declaraciones engañosas, ni con crítica destructiva, ni con acusaciones directas, ni con infundíos, mucho menos con cuestionamientos dolosos. Si el lector leyó con detenimiento el objetivo que aquél planteó en su supuesta réplica (A partir de lo señalado anteriormente, el objetivo del presente artículo es justificar la viabilidad del uso de una misma base de datos en diferentes productos de investigación), es por demás importante aclarar que nuestro artículo de 2017 no se centró en ese objetivo; por el contrario, se centró en, citaremos en extenso:

Revisar tres reportes de investigación publicados entre 2012 y 2013 por José Concepción Gaxiola Romero [...] en tres revistas especializadas [...] Dicha revisión comprende un análisis detallado de los tres reportes de investigación, cuyo propósito es discutir si los mismos se pueden considerar como corrupción académica (Piña, 2017; p. 238).

Note el lector que en ningún momento planteamos si o no era viable usar una misma base de datos en diferentes investigaciones. Primera pregunta elemental: ¿por qué Gaxiola no centró su réplica en lo que analizamos un año antes, esto es, los auto-plagios y la fabricación y/o falsificación de los datos? Pregunta que tuvo su primera No-R.

5. En los escritos empleados por Piña (2017), además de que se presentan resultados diferentes de una misma investigación, no se comprueba ninguna alteración o vicio en el desarrollo de los textos. A lo largo de la presente réplica se presentarán ejemplos de investigaciones que derivan de manera natural de diferentes productos de investigación empleando el uso de la misma base de datos. Es importante recalcar que los argumentos presentados son para aclarar ante la opinión pública y científica los señalamientos realizados por Piña sobre el trabajo realizado por nuestro equipo de investigación, no para intentar un debate con dicho autor, lo que sería infructuoso en términos científicos (Gaxiola, 2018; p. 109).

Ya que Gaxiola sostiene que presentará argumentos con fines de posicionarse ante la opinión pública y cien-

2 Sobre el respecto, le pedimos tenga presente esta última parte y la coteje con el contenido que se describe en la Tabla 5. tífica, le demostraremos que falta a la verdad. Así, si el lector tomó nota de la lectura de la cita textual, los elementos medulares en esta son dos: que no comprobamos que en los tres estudios analizados se hubiese alterado o viciado el desarrollo de los textos, y que, asimismo, se podía usar una misma base de datos para llevar al cabo diferentes estudios. Pues bien, en principio debemos mencionar que esos supuestos argumentos son usados injustificadamente $-\mathrm{y}$, por qué no decirlo, tramposamente- por Gaxiola, porque si bien el primero toca muy ligeramente los dos asuntos medulares sobre lo que publicamos en 2017, en su réplica curiosamente no desmintió nuestros dichos. Si el lector no lo ha hecho y se toma la molestia de leer nuestro artículo publicado en la revista Enseñanza e Investigación en Psicología, encontrará que al revisar minuciosamente tres artículos que entre 2012 y 2013 publicaron Gaxiola y colaboradores (en las Revistas de Psicología de la Pontificia Universidad Católica del Perú [PUCP], Colombiana de Psicología e Interamericana de Psicología), podrá constatar que al menos una veintena de párrafos eran iguales —o con ligeras modificaciones-, por lo que por razones menos que obvias, cuando alguien incurre en el auto-plagio, por supuesto que está viciando el desarrollo de los textos. ¿Insistimos, por qué en su supuesta réplica Gaxiola no hizo mención de los auto-plagios en más de una veintena de párrafos?

Nos permitimos hacer del conocimiento del lector que, aun cuando esos párrafos fueron citados textualmente en nuestro artículo de 2017, para evitar supuestas "malas", "engañosas" y "dolosas" interpretaciones, recientemente efectuamos un análisis apoyándonos en el programa Turnitin (https://turnitin.com/login_page.asp?lang=es), el cual fue desarrollado con el objeto de identificar plagios y auto-plagios en las publicaciones. Por tanto, nos dimos a la tarea de comparar las tres publicaciones analizadas en 2017, por ejemplo, iniciando con la de la Revista de Psicología de la PUCP (Gaxiola, González, Contreras y Gaxiola, 2012) y la de la Revista Interamericana de Psicología (Gaxiola, González, Domínguez y Gaxiola, 2013), cuyo informe de "originalidad" arrojó el 48\% de "similitud", distribuido de la siguiente manera:

1. El $38 \%$ corresponde al trabajo que apareció un año antes en la Revista de Psicología de la PUCP.

2. El $6 \%$ corresponde a fuente de la Internet (ResearchGate).

3. El $5 \%$ a la Revista Colombiana de Psicología de 2013.

Al hacer lo propio con los trabajos que aparecieron en la Revista de Psicología de la PUCP y en la Revista Colombiana de Psicología (Gaxiola, González y Gaxio- 
la, 2013), el informe de "originalidad" arrojó el 38\% de "similitud", distribuido de la siguiente manera:

1. El $30 \%$ corresponde a trabajo que apareció un año antes en la Revista de Psicología de la PUPC.

2. El $5 \%$ a fuente de la Internet (en RedAlyc).

3. El $3 \%$ a fuente de la Internet (journals.fcla.edu).

El lector seguramente entenderá que estamos haciendo mención de porcentajes elevados, del 48 y $38 \%$, sobre los cuales Gaxiola no comentó absolutamente nada en su supuesta réplica. Pero, además nos parece oportuno añadir que esa no ha sido la única ocasión en que aquél y sus colaboradores han incurrido en la práctica del auto-plagio. Le pedimos al lector que confirme nuestras afirmaciones con la lectura del contenido de la Tabla 1 (ver en el anexo), que incluye dos capítulos publicados por Gaxiola en colaboración con Martha Frías Armenta $^{3}$, el primero en un libro editado por la propia Universidad de Sonora y el segundo incluido en las Memoria en Extenso de la Asociación Mexicana de Psicología Social (AMEPSO), ambos de 2010. Nótese que se tratan en la mayoría de los casos de los mismos párrafos, del mismo objetivo, de la misma muestra, de los mismos análisis estadísticos, de los mismos resultados y de prácticamente las mismas conclusiones. Segunda pregunta elemental: ¿no constituye ello un auto-plagio?

A propósito de los auto-plagios, nos permitimos citar en extenso un párrafo de Koepsell y Ruiz de Chávez, obra que fue coeditada conjuntamente por la Comisión Nacional de Bioética en México (CONBIOÉTICA) y el Consejo Nacional de Ciencia y Tecnología (CONACYT):

Hay otra forma de plagio que también es dañina para la ciencia: el llamado auto-plagio. Esto es el acto de tomar las propias palabras de publicaciones anteriores y volver a publicarlas en otra obra sin referir correctamente su origen. Aunque no se tomen créditos por las expresiones de otros, en el auto-plagio los riesgos y los daños a la ciencia todavía están presentes. El motivo detrás de esta práctica yace claramente en las expectativas institucionales y recompensas asociadas con el número y el impacto de las publicaciones, así como el índice $h$, que ayuda a medir el impacto de un científico en un campo (Koepsell y Ruiz de Chávez, 2015; p. 60).

Prosiguiendo, no sólo estamos ante dos ejemplos claros de auto-plagios, sino que también nos parece extraño que Gaxiola haya practicado otra No-R en su su-

3 Profesora-Investigadora de Tiempo Completo en el Programa Docente de Derecho de la Universidad de Sonora (UniSon), además de ser Profesor con Perfil Deseable PRODEP e Investigadora Nacional nivel III. puesta réplica, al pasar por alto una valiosa oportunidad de desmentir sí o no había fabricado y/o falsificado deliberadamente los datos en dos de los tres trabajos analizados por Piña (2017), cuando aseguró que... además de que se presentan resultados diferentes de una misma investigación, no se comprueba ninguna alteración o vicio en el desarrollo de los textos. Por supuesto que Gaxiola falta a la verdad, ya que al analizar los contenidos de los datos incluidos en dos pares de Figuras que aparecen en las Revistas de Psicología de la PUPC (Reporte de investigación 1) e Interamericana de Psicología (Reporte de investigación 3), al comparar a dos grupos de estudiantes (no resilientes vs. Ios resilientes) concluimos que era inadmisible que en varios de los indicadores de bondad del ajuste ( $x 2$, grados de libertad, $p$, $\mathrm{BBNFI}, \mathrm{BBNNFI}, \mathrm{CFI}$ y RMSEA) los resultados fuesen en algunos casos los mismos y en otros presentaran diferencias más acusadas; todavía más inadmisible fue que los pesos factoriales fuesen en algunos casos iguales y en otros presentaran ligeras variaciones de -.3 ó de .3.

Con el objeto de que se pueda entender mejor sobre esto último, si los tres trabajos analizados contemplaron a la misma muestra ( $N=120$ estudiantes de bachillerato), si en los dos que incluyeron las figuras referidas (Revistas de Psicología de la PUPC e Interamericana de Psicología) se plantearon los mismos objetivos, si se reportó el uso de los mismos instrumentos y, además, si se corrieron los mismos análisis estadísticos, la pregunta más elemental que podemos plantear es la siguiente: ¿cómo alguien puede reportar valores iguales o con variaciones con la misma muestra y los mismos análisis estadísticos, si no es que se alteró o vició el desarrollo del texto? (Tabla 2, ver en anexo)

Entonces, ¿cómo es que los grupos de estudiantes no-resilientes obtuvieron los mismos indicadores de bondad del ajuste, pero no los del grupo de estudiantes resilientes? ¿Cómo explica Gaxiola que los primeros indicadores fueron iguales y luego distintos, si eran los mismos estudiantes, se planteó el mismo objetivo (y por tanto, las posibles relaciones entre factores), se usaron los mismos instrumentos y se corrieron los mismos análisis estadísticos? Y qué decir de los valores de los pesos factoriales, poniéndose de manifiesto cómo es que se fabricaron y/o falsificaron los datos deliberadamente, lo que se confirma con los valores incluidos de -.25 en el grupo "resiliente" en el Reporte 3, a pesar de que las relaciones de resiliencia con metas educativas y de estas con rendimiento se mantuvieron inalterados en los dos grupos de estudiantes. Sobre esta segunda parte de nuestro análisis en el trabajo de Piña (2017), Gaxiola 
tampoco replicó o desmintió nada y, por consiguiente, siguió practicando la No-R.

Citaremos, a propósito del manejo espurio de los datos, a Koepsell y Ruiz de Chávez:

En ocasiones, al desarrollar una hipótesis o teoría, los científicos no logran desprenderse de sus propios intereses y pueden pasar por alto algunos datos o incluso adecuarlos de tal forma que se ajusten mejor a su visión de cómo el experimento debe resultar; en el peor de los casos pueden manipular o tergiversar los datos de forma consciente para tratar de engañar al resto de la comunidad científica por las razones que sean (Koepsell y Ruiz de Chávez, 2015; p. 32).

6. Para finalizar, y retomando nuevamente el Código de Ética del Psicólogo (Sociedad Mexicana de Psicología, 2007), no se ofrece ningún comentario respecto a la violación de la ética por el uso de la base de datos en diferentes productos de investigación.

Una interpretación a modo del contenido de dicho artículo, no tenemos la menor duda. Ciertamente, no se señala nada sobre el respecto, aun cuando podría ser considerada como una conducta éticamente inaceptable (Aluja y Birke, 2004), lo que Ilamamos la "sobre-explotación de la muestra" (Piña, en revisión). En efecto, como se verá, hay 20 ó más productos de investigación reportados con cinco muestras distintas: de $\mathrm{N}=300$ madres de familia se contabilizan 3 (dos artículos en revista y un libro); de $\mathrm{N}=120$ estudiantes de bachillerato (incluidos los tres reportes analizados por nosotros en 2017) se contabilizan 4 productos; de $\mathrm{N}=240$ estudiantes de bachiIlerato se contabilizan 2; de $\mathrm{N}=182$ madres de familia se contabilizan 7 (incluidos los dos capítulos auto-plagiados, referidos en la Tabla 1); finalmente, y a la espera de que aparezcan otras más, de $\mathrm{N}=254$ madres de familia se contabilizan 7 . El lector, quien no es para nada ingenuo como nos deja entrever Gaxiola, sabe que la "sobre-explotación de la muestra" sólo se puede dar cuando se plantean objetivos iguales o muy parecidos, matizados con ciertas frases por aquí o por allá, y sobre todo, cuando de manera conveniente (demasiado conveniente, diríamos) se analizan unas variables y luego otras.

Para demostrar nuestras afirmaciones, le pedimos al lector que por favor revise minuciosamente el contenido de los objetivos de varios de los productos de investigación de Gaxiola y colaboradores, que aparecen en la Tabla 3 (Ver en el anexo)

Retomando el contenido de los puntos 4 (A lo largo de la presente réplica se presentarán ejemplos de investigaciones que derivan de manera natural diferentes productos de investigación empleando el uso de la misma base de datos) y 5 (no se ofrece ningún comentario respecto a la violación de la ética por el uso de la base de datos en diferentes productos de investigación) planteados por Gaxiola, y aceptando sin conceder la interpretación a modo que hace de uno de los artículos del Código de Ética del Psicólogo, usar cinco muestras presumiblemente distintas y generar más de 20 productos de investigación, que se basan en los mismos o en objetivos bastante similares, incluyendo ciertos factores en unas y eliminándolos en otras, sólo evidencia que la "sobre-explotación de la muestra" ha sido una práctica recurrente en aquél y sus colaboradores. Por cierto, una cosa es re-evaluar el resultado de una investigación para los fines de comprobar o refutar los hallazgos, y otra muy distinta es que de una investigación, como por ejemplo, la Tesis Doctoral de Gaxiola (defendida en 2007 con la dirección de Martha Frías Armenta), se tengan al día de hoy registrados siete productos supuestamente diferentes (le recuerdo al lector que los dos capítulos descritos en la Tabla 1 forman parte de esta serie de trabajos publicados con la muestra de 182 madres de familia). Sobre el respecto Gaxiola no hizo pronunciamiento alguno en su supuesta réplica; otro ejemplo más de la No-R. Revísese, por favor, el contenido del punto 6 .

7. En el artículo 58 del citado código se indica: "El psicólogo no publica como datos originales, aqueIlos que ya hayan publicado previamente. Esto no impide republicar datos cuando vayan acompañados del reconocimiento o permiso apropiado" (p. 63). Aquí no se aclara si por "datos" se incluyen también las características de la muestra y de los instrumentos, porque, si es así, ninguno de los ejemplos expuestos en la sección anterior, donde se amplifica el uso de los mismos resultados en diferentes productos, sería ético o, en otras palabras, corrupción académica, tal y como afirma Piña (2017) [...] El problema que se presenta y que puede dar lugar a suspicacias de los críticos es que en ocasiones no es posible especificar de dónde provienen los datos de la muestra y los instrumentos empleados cuando las fuentes aún no se encuentran publicadas, sobre todo al momento en que se someten a distintas revistas dos o más trabajos con distintos resultados de la misma muestra. Es importante aclarar que, en ocasiones, no es posible referir que los datos se usaron en diferentes productos académicos porque las revisiones de los artículos en diversas revistas se llevan a cabo de manera paralela y, como ocasionalmente 
sucede, no todos los productos son aceptados con el tiempo suficiente para aclarar cuál fue el primer reporte de investigación procedente de los datos reportados (Gaxiola, 2018; p. 112).

Gaxiola falta de nuevo a la verdad, porque basta y sobra que el lector contabilice los casos en que se ha incurrido en auto-plagios, en la fabricación y/o falsificación de los datos, así como en su presentación por partida doble. Por cierto, lo interesante es que en ningún lugar de los trabajos publicados por aquél y sus colaboradores, se... acompaña el reconocimiento o permiso apropiado correspondiente. Es decir, en ninguno de los productos hacen mención de algo que cualquier investigador se obliga a hacer: el presente trabajo constituye la versión extensa de una presentada en tal o cual evento; o esta otra: el presente trabajo deriva de la Tesis Doctoral del primer autor, dirigida por el segundo autor. Extraño que Gaxiola haga uso de un artículo del Código de Ética del Psicólogo, el cual por supuesto que viola flagrantemente.

Pero, lo que resulta todavía más interesante de la cita textual previa, es el contenido de la parte final: Es importante aclarar que, en ocasiones, no es posible referir que los datos se usaron en diferentes productos académicos porque las revisiones de los artículos en diversas revistas se llevan a cabo de manera paralela y, como ocasionalmente sucede, no todos los productos son aceptados con el tiempo suficiente para aclarar cuál fue el primer reporte de investigación procedente de los datos reportados. Es decir, resulta que un investigador en ocasiones — sólo en algunas - no sabe de dónde se obtuvieron los datos de la muestra y de los instrumentos que ha usado; bastante extraño de alguien quien, junto con sus colaboradores, ha usado cinco muestras distintas y llevan 20 ó más productos de investigación reportados. Le demostraremos a Gaxiola por qué falta a la verdad de nueva cuenta. El lector constatará, revisando el contenido de la Tabla 4, que entre la aceptación del reporte 1 (25 de octubre de 2011 en la Revista de Psicología de la PUPC) y el envío del reporte 3 (17 de abril de 2012 en la Revista Interamericana de Psicología) pasaron más de 180 días. Si los reportes 1 y 3, analizados por Piña en 2017, representan dos claros ejemplos de párrafos auto-plagiados y de fabricación y/o falsificación deliberada de los datos (recuerde el lector el contenido de la Tabla 3), ¿cómo explica entonces Gaxiola que aceptado el Reporte 1 el 25 de octubre de 2011 y recibido el tercero el 17 de abril de 2012 no pudo haber tenido tiempo suficiente para aclarar cuál fue el primer reporte de investigación procedente de los datos reportados? Una extraña y no menos curiosa respuesta, por decir lo menos.

\section{Tabla 4.}

Fechas de recibido y de aceptación de tres reportes de investigación de Gaxiola y colaboradores.

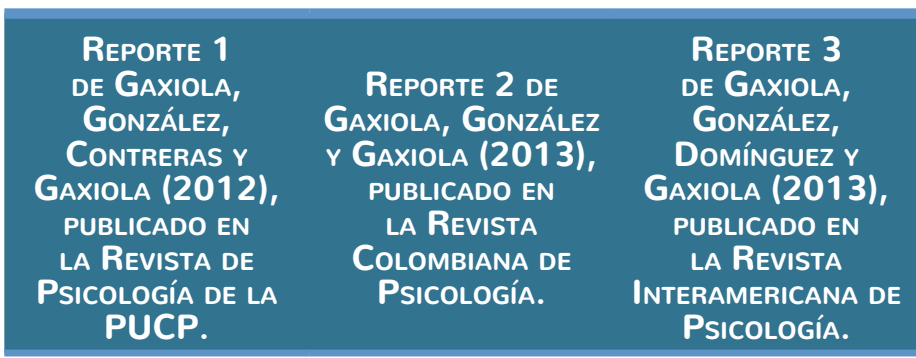

Recibido: 24 de junio de 2011

Recibido: 21 de

Recibido: 17 de

Aceptado: 25 de octubre de 2011 junio de 2011 abril de 2012

Aceptado: 3 de mayo de 2013

Aceptado: 8 de mayo de 2013

Y con relación a la sui generis justificación que hace sobre el uso de instrumentos en la cita textual anterior (Número 6), con motivo del artículo de Piña (2018), en el que realizamos una exhaustiva revisión de 17 trabajos publicados (en revistas arbitradas, Memorias en Extenso, capítulos en libros o libros) por Gaxiola y colaboradores, demostramos de manera fehaciente que en 15 de los 17 reportaron el uso de diferentes instrumentos de medida (nombrados indistintamente como escalas, inventarios o cuestionarios, en varios trabajos inclusive usando de manera intercambiable los términos), sin que se haya encontrado la evidencia tangible y por escrito, tal y como marcan los cánones en la materia, de los artículos de validación correspondientes de todos y cada uno de los instrumentos de medida usados. Claro está que cuando se reportan datos o se hacen menciones de contenidos en citas, instrumentos y resultados de investigaciones de manera no ética, lo que se comprueba es que justamente se está alterando o viciando el desarrollo de los textos.

Por ejemplo, junto con la fabricación y/o falsificación deliberada de los datos, así como su uso repetido en pares de investigaciones, en la Tesis Doctoral de 2007 defendida por Gaxiola, que se publicó como libro siete años después por Gaxiola, Frías y Corral (2014), nos podemos encontrar la alteración o vicio en el desarrollo de los textos, en el uso no ético de datos y referencias, aparentando por consiguiente que el libro de 2014 derivó de un estudio original y que contenía datos, cifras, referencias y demás, todos supuestamente originales (Tabla 5, ver en el anexo).

Entre la Tesis Doctoral de 2007 y el libro de 2014, lo único diferente fue que en este se incluyó un prólogo (p. 11), que consta de una página y unos pocos párrafos, con algunas ligeras modificaciones otros párrafos a lo largo del libro (los que hay, seguro fueron debido al pro- 
ceso editorial). Asegurar, asimismo, que en el libro aparecen actualizaciones de antecedentes y/o conclusiones derivadas de hallazgos nuevos, es faltar a la verdad, pues el lector puede confirmar justo lo contrario con la lectura de más de una veintena de párrafos seleccionados de los diferentes apartados, donde el contenido es igual. Adicionalmente, considerando el contenido de la Tesis Doctoral y del libro, por supuesto que la diferencia de siete años entre una y el otro, es natural que los datos, tanto los que corresponden a la muestra, sus características socio-demográficas, los derivados de los análisis estadísticos... pierden vigencia a los siete años; de otra suerte, ¿entonces, lo psicológico y social se mantienen invariantes a lo largo del tiempo y entre situaciones?

Nos permitimos citar de nuevo a Koepsell y Ruiz de Chávez, sobre el manejo de las fuentes y citas:

Un registro claro de datos no es una cuestión de cortesía o de reconocimiento al trabajo de otros, sino que es un medio para comprobar y refinar las hipótesis y teorías sobre la base de las observaciones realizadas en el pasado. Omitir este registro hace un flaco favor a la comunidad científica y entra en conflicto con el ethos de la ciencia. El avance de la ciencia depende de la interacción de una comunidad, por ello es importante que se faciliten las fuentes y se contabilicen con exactitud los medios, el lugar y la hora de cada estudio (Koepsell y Ruiz de Chávez, 2015; p. 34).

Por tanto, si Gaxiola pretende hacer pasar un libro como un producto novedoso —que se insiste, derivó y es la calca de la Tesis Doctoral-, sería interesante que aclarara públicamente por qué en los apartados de introducción, en los primeros párrafos de la Tesis Doctoral y del libro se cambió la referencia: en la primera aparece la OMS y en el segundo una de la Secretaría de Salud. ¿No es acaso ello un ejemplo conspicuo de alterar o viciar el desarrollo de los textos? Lo mismo se aplicaría en el caso de la cita del INEGI y los datos que la amparan en el apartado de participantes, pues en la Tesis Doctoral la cita corresponde a datos de 2000 (INEGI, 2001) y en el libro a datos de 2010 (INEGI, 2010). ¿Cómo puede ser posible ello si se trató de la misma muestra? ¿O sea, cómo se explica que en 2000 había en el estado de Sonora 2.6 hijos por familia y, siendo la misma muestra, para el año 2010 ya se había reducido el promedio a 2.3 hijos por familia? Por supuesto que se alteró y vició el desarrollo de los textos, aun cuando Gaxiola pretenda hacer ver al lector lo contrario. Cualquier investigador sabe perfectamente $y$, digamos que es una suerte de convención, que un recién titulado de Maestría o Doctorado publique una versión de su tesis teniendo como coautor a su director(a) de tesis, como por ejemplo en Castro, Matamoros-Tapia, Mijangos, Nava-Guerrero y Sánchez-Sosa (2012; p. 78), donde se especificó lo siguiente: Una versión parcial y preliminar del estudio constituyó la tesis doctoral del primer autor bajo la dirección del último.

Adicionalmente, entre la Tesis Doctoral y el libro de marras hay otro asunto que por ningún motivo puede ser pasado por alto: el de la coautoría. En efecto, si alguien es autor de una Tesis Doctoral, la escribe, la defiende y con ella obtiene un grado académico " $x$ ", está claro que, en caso de que posteriormente se publique como libro, quien la escribió y defendió debería ser el único autor; nada justifica que en este caso, la directora de la tesis (Martha Frías Armenta) y otra persona (Víctor Corral Verdugo), figuren como coautores de la tesis convertida en libro al paso de siete años. Aquí es oportuno citar de nuevo a Koepsell y Ruiz de Chávez, a propósito del asunto de la autoría:

El primer deber de la publicación científica es la verdad. Como ya se mencionó anteriormente, la ciencia opera en parte bajo el supuesto de una forma de realismo en el que creemos que en la naturaleza subyacen leyes cognoscibles, consistentes y universales, y que el objeto de la ciencia es mejorar la comprensión del funcionamiento de la naturaleza [...] Un artículo científico implica no sólo al autor, sino también co-autores, compañeros de investigación, organismos de financiación, instituciones que albergan el estudio, así como a la comunidad científica y al público en general. Dada la red de interlocutores, es natural que las diversas funciones se hayan vuelto cada vez más complicadas y no sorprende que una serie de malos actos que se han hecho públicos, así como perjuicios a la ciencia, sean el resultado de no considerar y cumplir con los deberes de la autoría (Koepsell y Ruiz de Chávez, 2015; pp. 47-48).

Ocupar una posición de autoridad no es suficiente. Simplemente quien estar a cargo de quienes escriben un documento es insuficiente para justificar una autoría, a pesar de los lineamientos institucionales o prácticas disciplinarias; debe haber una contribución intelectual al trabajo. La simple lectura de un documento también es insuficiente; cada autor debe, cuando menos, leer el trabajo que se le atribuye: los correctores y los editores no son autores [...] (Koepsell y Ruiz de Chávez, 2015; pp. 52-53). 
¿Quién escribió y defendió la Tesis Doctoral? Si hay un único autor y a la vuelta de siete largos años aparece la tesis en la forma de libro, incluyendo a dos co-autores, que no aportaron nada adicional a este, tal y como ha quedado comprobado con el extenso contenido de la Tabla 5 (donde, se insiste, no sólo es prácticamente lo mismo que en la tesis, con alteraciones y vicios de fuentes y citas), ¿qué justifica entonces la coautoría tanto de Martha Frías Armenta y Víctor Corral Verdugo en el libro publicado extemporáneamente?

Además, es incorrecto como asegura Gaxiola en el punto 6, que en ocasiones - le recordamos al lector, que sólo en algunas- no es posible dar cuenta de la fuente, en particular cuando todavía no se publica el trabajo que los contenga. Falta a la verdad, puesto que en ese último artículo publicado por nosotros (Piña, 2018), fue moneda corriente que él y sus colaboradores se apoyaran en las siguientes leyendas (resaltadas con negritas y en cursivas), para lo cual me referiré al mismo instrumento, usado en diferentes reportes de investigación que incluyeron a la misma muestra:

1. Bienestar psicológico. Reportado en investigaciones cuya muestra fue de 254 madres de familia, de las cuales se mencionan algunas por año de aparición: Gaxiola, Gaxiola, González y Valenzuela (2014; p. 558), Gaxiola, Gaxiola, Valenzuela y Aranda (2014; p. 979), Gaxiola (2016; p. 52), Gaxiola, Millanes y Aranda (2017; p. 29). En estos y otros reportes más se afirma que se trató de un inventario de 12 ítems desarrollado para el estudio. En todos se afirmó que en el estudio piloto se obtuvo un alfa de 0.88 . Es decir, no logramos comprender cómo es que el Dr. Gaxiola Romero, quien supuestamente argumentó que se podía hacer uso de la misma muestra para presentar diferentes productos si contenían objetivos diferentes (recuerde el lector el contenido de la Tabla 5), señale que entre 2014 y 2017, para cada uno de los estudios se desarrolló un inventario conformado por 12 ítems. O sea, ¿desarrolló un inventario para medir bienestar psicológico en cada uno de los siete productos que se han contabilizado con la muestra de 254 madres de familia? La verdad, dicha sea de paso, no entendemos. Adicionalmente, nos parece por demás necesario y oportuno hacer de dominio público lo siguiente, de algo que ya se mencionó una parte como nota extensa al pie de página en el artículo de Piña (2018):

Revisando otros trabajos publicados por varios de los colaboradores de Gaxiola Romero, encontra- mos que en uno de ellos, coautoría de González y Rivera (2016; p. 39), que es el capítulo 2 de un libro coordinado justo por Gaxiola y Palomares, se lee que: Escala de Bienestar Psicológico. El bienestar psicológico se evaluó con 12 ítems que fueron desarrollados por Gaxiola, Gaxiola, González y Valenzuela (2014), que se basan en el inventario de Ryff (1989), y por lo tanto, sus reactivos evalúan las áreas de autonomía, relaciones positivas, propósitos de vida, autoaceptación, dominio del entorno y crecimiento personal. Esta referencia es de un trabajo que se presentó ese mismo año, como ponencia, en el Congreso Mexicano de Psicología, organizado por la Sociedad Mexicana de Psicología.

En la búsqueda de la memoria en extenso, disponible en la Internet ${ }^{4}$, en las páginas 557 a la 559 aparece la ponencia de marras, sólo que con la siguiente salvedad, a saber, que en la página 558 se lee: El bienestar psicológico fue medido con un inventario desarrollado para el estudio, basado en el inventario Ryff (1989), donde se incluyeron preguntas sobre autonomía, relaciones positivas, propósito en la vida, autoaceptación, dominio del entorno y crecimiento personal... Por tanto, nótese que la "fuente" a la que hacen mención González y Rivera no incluyó una escala desarroIlada por los cuatro autores, quienes simplemente se limitaron a decir que fue un "inventario desarrollado para el estudio". Finalmente, en la página 41, en la Tabla 3, González y Rivera señalan que el alfa de la escala fue de 0.88 .

El problema, es que en el mismo libro, pero en su capítulo 3, Aranda y Frías (2016; p. 71) mencionan que utilizaron una escala de bienestar personal, desarrollada por González y Gaxiola (s.f), que consta de 11 ítems cuyo valor del alfa de Chronbach en la prueba piloto fue de 0.88. Nótese que en cualquier caso, esto es, sean 11 ó 12 preguntas, el valor del alfa para la escala o inventario fue el mismo, de 0.88 , con la salvedad de que los autores de ambos capítulos refieren una fuente distinta: en el primero de los casos se trató de una ponencia presentada en un Congreso Nacional de Psicología, en tanto que la segunda es sin fecha e incluye a dos de los cuatro autores que participaron en esa ponencia. Por consiguiente, si el lector toma nota de lo escrito en el resumen, en ningún momento

4 http://psicologia.mx/images/sampledata/XXII_ CMP/MemoriaXXII.pdf 
se llevó a cabo ningún análisis de validación de la escala de Bienestar Psicológico, utilizando los autores la expresión a la que se ha recurrido una y otra vez, es decir, "en un estudio piloto..."

Finalmente, ¿cuál escala o inventario, supuestamente "validado", se debe tomar como

referencia? ¿La que se asegura se elaboró para cada uno de los siete estudios que incluyeron a la misma muestra de $\mathrm{N}=254$ madres de familia? ¿La de Gaxiola, Gaxiola, González y Valenzuela (2014)? ¿O la de González y Gaxiola (s.f.)? Pero, no se puede omitir el hecho de que dos de los cuatro colaboradores de la ponencia presentada en 2014 aparecen como coautores de una ponencia registrada en el Libro de Resúmenes del Congreso Euro-Latinoamericano de Intervención en Justicia Juvenil, que se celebró en Almería, España, en 2015; en dicho Libro de Resúmenes, en la página 18 , se encuentra la ponencia intitulada Construcción y Validación de una Escala de Bienestar Subjetivo, coautoría de Aranda, Frías, González, Valenzuela y Barrios (2015), en cuyo resumen se lee: Se aplicó la escala a 227 estudiantes de una escuela secundaria pública en Sonora, México. Posteriormente, se analizó la confiabilidad de la escala resultando un alfa de Cronbach $>.60$. Su validez de contenido fue por paneles de expertos y para la validez de constructo se utilizó el análisis factorial confirmatorio de segundo orden. Los resultados revelaron que el instrumento es confiable ya que tiene un alfa de .88; de igual manera, el análisis factorial confirmatorio indicó que el modelo propuesto de dos factores no se ajustaba a los por lo que se optó por dejar un factor [...]

2. Estilos de crianza o adaptabilidad. Reportado originalmente en la Tesis Doctoral de Gaxiola y en investigaciones, cuya muestra fue de 182 madres de familia y de las cuales se mencionan algunas por año de aparición: Gaxiola (Tesis doctoral defendida en 2007; p. 83), Gaxiola y Frías (2007; p. 222), Gaxiola y Frías (2008; p. 21), Gaxiola y Frías (2012; p. 265), Gaxiola, Frías y Corral (2014; pp. 102-103). En estos y otros reportes más se afirmó que se trató de un inventario o escala que consta de 132 preguntas, elaborado(a) para la investigación por Gaxiola y Ribes (no publicado). ¿Se refiere al Dr. Emilio Ribes Iñesta, el prestigiado y reconocido investigador mexicano que labora en la Universidad Veracruzana? Todos en este país sabemos perfectamente que el Dr. Ribes Iñesta no se dedica a tareas relacionadas con la "elaboración" y "validación" de instrumentos de papel y lápiz, de manera tal que no fue coautor de la supuesta elaboración del inventario o escala de marras. Sólo que, nos permitimos añadir, que en nuestro trabajo publicado en 2018 (Piña, 2018) comentamos que a mediados de la pasada década, Gaxiola, Frías, Cuamba, Franco y Olivas (2016) adaptaron y validaron un inventario originalmente diseñado y validado en los Estados Unidos de Norteamérica por Robinson, Mandleco, Frost y Hart (1995), que constaba de 133 preguntas, el cual en el proceso de análisis

y depuración estadísticas incluyó un total de 62 preguntas $^{5}$. Esta misma cantidad fue la que sometieron a validación Gaxiola y colaboradores, que ha sido reportada en un estudio con 132 madres de familia por Gaxiola y Frías (2010c; pp. 110-111), así como en su versión de 11 preguntas (denominada como Escala de Estilos de Crianza Autoritativo) en otros estudios, como por ejemplo Gaxiola, Millanes y Aranda (2017; p. 28) y Gaxiola, Gaxiola y Frías (2017; p. 5). Sin embargo, es importante mencionar que el referido Estilo de Crianza Autoritativo, incluso en la versión validada por Gaxiola y colaboradores, consta de seis factores, a saber: envolvimiento (11 preguntas), razonamiento (7 preguntas), participación democrática (5 preguntas), relación amistosa (4 preguntas), falta de supervisión (6 preguntas) e ignorar el mal comportamiento (4 preguntas), que sumadas dan en total 37 preguntas, con el añadido de que estos dos últimos factores pertenecían al Estilo de Crianza Permisivo, que terminaron agrupándose en de Crianza Autoritativo. ¿Por qué hacemos mención de todo ello? Por la simple y sencilla razón de que no existe un Estilo de Crianza Autoritativo conformado por 11 preguntas, sino en todo caso un factor particular dentro de este, que a juzgar por una de las preguntas utilizadas (Le dice las cosas que hace bien), formarían parte del factor llamado envolvimiento.

3. Algo sin duda digno de mención y merecedor de una amplia discusión, es lo siguiente, que hay que leer textualmente para que no se nos acuse de falsear la información o proferir infundíos y demás:_Gaxiola (2016; p. 53): Los instrumentos

$5 \quad$ La escala original de Robinson et al. (1993), evalúa tres estilos de crianza parental: autoritativo (27 preguntas distribuidas en cuatro factores), autoritario (20 preguntas distribuidas en cuatro factores) y permisivo (15 preguntas distribuidas en tres factores), que sumadas dan como total 62 preguntas. 
que se encontraban en inglés fueron traducidos al español por expertos y se realizó un estudio piloto previo en una población similar a la del presente estudio. En éste los instrumentos obtuvieron valores de Alfa aceptables ( $>0.60$ ), y se les realizó análisis factorial confirmatorio para medir su validez de constructo. Si lo que afirma aquél es veraz y por supuesto comprobable, ¿en dónde están publicados todos los estudios de validación de cada uno de los instrumentos que ha reportado él solo o en conjunto con otros colaboradores? La lectura de nuestro artículo de 2018 revelará que esos estudios siguen siendo al día de hoy un enorme misterio. Pero, queremos reparar en un asunto que también revela la gravedad de las faltas a la verdad por parte de Gaxiola; a saber, que en esa publicación de 2016, resaltada con negritas, se hace mención del inventario intitulado bienestar psicológico, del que se dice fue desarrollado para la presente investigación. Si este inventario se basó en la propuesta de Ryff, ¿cómo se puede afirmar que se tradujo y validó, para luego escribir que fue desarrollado para el estudio en varias ocasiones, tal y como se puede constatar con lo escrito en párrafos previos?

$Y$ respecto del manejo y reporte de fuentes y citas, citaremos de nuevo en extenso a Koepsell y Ruiz de Chávez:

En ocasiones, al desarrollar una hipótesis o teoría, los científicos no logran desprenderse de sus propios intereses y pueden pasar por alto algunos datos o incluso adecuarlos de tal forma que se ajusten mejor a su visión de cómo el experimento debe resultar; en el peor de los casos pueden manipular o tergiversar los datos de forma consciente para tratar de engañar al resto de la comunidad científica por las razones que sean (Koepsell y Ruiz de Chávez, 2015; p. 32).

¿Comentarios negativos? ¿Declaraciones engañosas? ¿Crítica destructiva? ¿Acusaciones directas? ¿Infundíos? ¿Cuestionamientos dolosos? ¿Pues qué parte de la enorme y sustentada evidencia presentada en 2017, 2018 y aquí no ven el Gaxiola y sus colaboradores? Nos parece, expuesto lo anterior, que a este bien podría aplicárseles la Ley de Puder, que a la letra dice:

Cualquier cosa que empieza bien, acaba mal. Cualquier cosa que empieza mal, acaba peor. (Bloch, 1992; p. 19).
Así los hechos, el recuento es el siguiente:

1. Auto-plagios en múltiples párrafos, contenidos en varios artículos, Memorias en Extenso y libros.

2. Fabricación y alteración deliberada de datos.

3. Uso repetido de datos en pares de investigaciones, que también constituye un ejemplo de auto-plagio, en particular cuando nunca se hace mención de su uso previo en publicaciones con títulos distintos.

4. Citación de referencias inexistentes sobre los instrumentos que presumiblemente fueron validados.

5. Repetición de trabajos de investigación usando la misma muestra y con objetivos iguales o muy similares.

6. Hasta incluir como coautor al Dr. Emilio Ribes Iñesta, quien, sin temor a equivocarnos, podemos afirmar que nunca colaboró en tal calidad $y$, muchos menos, supuestamente validando el instrumento de marras.

El recuento y los elementos descritos en Tablas, citas textuales, etcétera, están ahí y confiamos que el lector -quien por supuesto no es ingenuo como nos hace saber Gaxiola- constatará todo lo que antes y hasta aquí hemos afirmado, con datos duros y evidencia, tal y como marcan los cánones en la ciencia. Es tiempo de que las autoridades académicas y administrativas en la Universidad de Sonora, así como también los editores/ directores de revistas y las propias del Sistema Nacional de Investigadores, pongan coto a la simulación y el engaño académico. Por lo demás, no se trata únicamente de Gaxiola, puesto que también han incurrido en conductas éticamente inaceptables y éticamente cuestionables (Aluja y Birke, 2004; Hirsch, 2012, 2016) varios Profesores-Investigadores y estudiantes de Licenciatura y Posgrado de la Universidad de Sonora.

Si no se atacan frontalmente todos los actos de simulación y engaño académico de Gaxiola y muchos de sus colaboradores, lo que se demostrará en los hechos es que se puede actuar impunemente y que tales actos inclusive seguirán siendo premiados con el paso de una categoría y nivel en la Universidad de Sonora, con Becas al Desempeño Académico, con promociones en el mismo Sistema Nacional de Investigadores y con la titulación de esos estudiantes bajo condiciones harto sospechosas, considerando su participación como coautores en muchos de los puntos contenidos en el recuento final.

En 2015, el Consejo Nacional de Ciencia y Tecnología, CONACYT, a través de quien fungía entonces 
como director General, Enrique Cabrero Mendoza, en la presentación del libro Ética de la investigación: Integridad científica, escribió lo siguiente:

Así, el Consejo Nacional de Ciencia y Tecnología junto con la Comisión Nacional de Bioética se honran en presentar esta guía para el quehacer científico, que será referente nacional en materia de ética e integridad científica. Esta obra es un reflejo del compromiso de ambas instituciones con el fortalecimiento de la práctica científica en México (Cabrero, 2015; p. 10).

¿Quién dará el primer paso? ¿El Consejo Nacional de Ciencia y Tecnología? ¿La Universidad de Sonora? ¿Los editores/directores Generales de las Revistas?

\section{Referencias}

Aluja, M. y Birke, A. (2004). Panorama general sobre los principios éticos aplicables a la investigación científica y la educación superior. En M. Aluja y A. Birke (Eds.), El papel de la ética en la investigación científica y la educación superior (pp. 87-143). México: Fondo de Cultura Económica/Academia Mexicana de Ciencias.

Aranda, C.L. y Frías, M. (2016). Apoyo social y ambiente familiar positivo relacionado con el bienestar subjetivo desde la perspectiva de los adolescentes. En J.C. Gaxiola y J. Palomar (Coords.), El bienestar psicológico: Una mirada desde Latinoamérica (pp. 61-88). Hermosillo, México: Qartuppi, S. de R.L. de C.V.

Aranda, C.L., Frías, M., González, S., Valenzuela, E.R. y Barrios, M.I. (2015). Construcción y validación de una escala de bienestar subjetivo. En J. García-García, F. ZaldívarBasurto, L. de la Fuente-Sánchez. E. Ortega-Campos, M.J. Gil-Fenoy, A.M. Pérez-Vallejo et al. (Comps.), Libro de Resúmenes del Congreso Euro-Latinoamericano de Intervención en Justicia Juvenil (p. 18). Almería, España: Universidad de Almería.

Bloch, A. (1992). El libro completo de las Leyes de Murphy. México: Diana.

Cabrero, E. (2015). Presentación. En Koepsell, D.R. y Ruiz de Chávez, M.H. (Autores), Ética de la investigación: Integridad científica (pp. 7-10). México: CONBIOÉTICA/ CONACYT.

Castro, C.G., Matamoros-Tapia, J.L., Mijangos, M.G., NavaGuerrero, E.N. y Sánchez-Sosa, J.J. (2012). Intervención conductual aguda en una sesión, en las enfermedades hipertensivas del embarazo: Un estudio piloto. Revista Latinoamericana de Medicina Conductual, 2(2), 78-86.

Frías, M., Rodríguez, I. y Gaxiola, J.C. (2003). Efectos conductuales y sociales de la violencia familiar en niños mexicanos. Revista de Psicología de la PUPC, XXI(1), 42-70.

Gaxiola, J.C. (2007). Factores protectores, estilos de crianza y maltrato infantil: Un modelo ecológico. Tesis para optar por el grado de Doctor en Ciencias Sociales, no publicada. Hermosillo, México: Universidad Autónoma de Sinaloa/ Universidad de Sonora.
Gaxiola, J.C. (2016). Adaptabilidad psicológica en madres e hijos con experiencias de violencia intrafamiliar. México: Universidad de Sonora/Pearson.

Gaxiola, J.C. (2018). Réplica a Julio A. Piña López: “Predictores del rendimiento académico de bachilleres, de Gaxiola y colaboradores: ¿Un ejemplo de corrupción académica? Enseñanza e Investigación en Psicología, 32(1), 108-114.

Gaxiola, J.C. y Frías, M. (2005). Las consecuencias del maltrato infantil: Un estudio con madres mexicanas. Revista Mexicana de Psicología, 22(2), 363-374.

Gaxiola, J.C. y Frías, M. (2007). Los factores protectores y la adaptabilidad al abuso infantil: un estudio con madres mexicanas. Revista Mexicana de Psicología, Número Especial (Octubre), 222-223.

Gaxiola, J.C. y Frías, M. (2008). Un modelo ecológico de factores protectores del abuso infantil: un estudio con madres mexicanas. Medio Ambiente y Comportamiento Humano, 9(1-2), 13-31.

Gaxiola, J.C. y Frías, M. (2010a). Determinantes de la calidad de vida en mujeres sobrevivientes del maltrato infantil. En A.L. Banda, J. Palomar y A. González (Comps.), Calidad de vida. Un enfoque psicológico (pp. 75-89). Hermosillo, México: Editorial de la Universidad de Sonora.

Gaxiola, J.C. y Frías, M. (2010b). Variables asociadas a la calidad de vida en mujeres sobrevivientes del maltrato infantil. En S. Rivera, R. Díaz-Loving, I. Reyes, R. Sánchez y L.M. Cruz (Eds.), La psicología social en México. Vol. XIII (pp. 791-796). México: Asociación Mexicana de Psicología Social.

Gaxiola, J.C. y Frías, M. (2010c). Child maltreatment, selfregulation, and parenting. En M. Frías y V. Corral (Eds.), Bio-psycho-social perspectives on interpersonal violence (pp. 101-121). Hauppauge, NY: Nova Science Publishers.

Gaxiola, J.C. y Frías, M. (2011). Consecuencias a largo plazo del maltrato infantil en madres mexicanas. Madrid: Editorial Académica Española.

Gaxiola, J.C. y Frías, M. (2012). Factores protectores, estilos de crianza y maltrato infantil: un modelo ecológico. Psyecology, 3(3), 259-270.

Gaxiola, J.C., Frías, M. y Corral, V. (2014). Ecología de los factores protectores y de riesgo de la crianza materna y el maltrato infantil. México: Clave Editorial.

Gaxiola,J.C., Frías, M.yFigueredo, A.J. (2011). Factores protectores y los estilos de crianza: un modelo bioecológico. Revista Mexicana de Investigación en Psicología Social y de la Salud, 1(1), 28-40.

Gaxiola, J.C. y Gaxiola, E. (2016). Apoyo social, resiliencia, bienestar psicológico y crianza en mujeres víctimas de violencia de pareja. En J. C. Gaxiola y J. Palomar L. (Coords.): El bienestar psicológico: Una mirada desde Latinoamérica (pp. 139-164). Hermosillo, México: Qartuppi, S. de R.L. de C.V.

Gaxiola, J.C., Gaxiola, E., Aranda, C.L. y Millanes, R.M. (2014). Trayectoria desadaptativa de la violencia de pareja, crianza autoritativa y maltrato infantil. En Memoria en Extenso del Congreso Mexicano de Psicología (pp. 559562). México: Sociedad Mexicana de Psicología/Colegio Mexicano de Profesionistas de Psicología. 
Gaxiola, J.C., Gaxiola, E. y Frías, M. (2017). Variables contextuales e individuales relacionadas con la crianza positiva materna. Universitas Psychologica, 16(2), 1-12.

Gaxiola, J.C., Gaxiola, E., González, S. y Valenzuela, E.R. (2014). Trayectoria adaptativa de la crianza positiva materna. En Memoria en Extenso del Congreso Mexicano de Psicología (pp. 557-559). México: Sociedad Mexicana de Psicología/ Colegio Mexicano de Profesionistas de Psicología.

Gaxiola, J.C., Gaxiola, E., Valenzuela, E.R. y Aranda, C.L. (2014). La crianza materna positiva: un modelo estructural de interdependencia funcional. En S. Rivera, R. Díaz-Loving, I. Reyes-Lagunes y M.M. Flores (Coords.): La Psicología Social en México, Vol. XV (pp. 977-983) México: Asociación Mexicana de Psicología Social.

Gaxiola, J.C., González, S. y Contreras, Z. (2010). Resiliencia, metas y contexto social: Su influencia en el rendimiento académico en estudiantes de preparatoria. En J. Angulo, A.A. Valdés, S.V. Mortis y R.I. García (Coords.), Educación, tecnología e innovación (cinco páginas). Ciudad Obregón, México: Instituto Tecnológico de Sonora.

Gaxiola, J.C., González, S. y Contreras, Z. (2012). Influencia de la resiliencia, metas y contexto social en el rendimiento académico de bachilleres. Revista Electrónica de Investigación Educativa, 14(1), 164-181.

Gaxiola, J.C., González, S., Contreras, H.Z. y Gaxiola, E. (2012). Predictores del rendimiento académico en adolescentes con disposiciones resilientes y no resilientes. Revista de Psicología de la PUCP, 30(1), 48-74.

Gaxiola, J.C., González, S., Domínguez, M. y Gaxiola, E. (2013). Autorregulación, metas y rendimiento académico en bachilleres con disposiciones resilientes y no resilientes. Revista Interamericana de Psicología, 48(1), 71-82.

Gaxiola, J.C., González, S. y Gaxiola, E. (2013). Autorregulación, resiliencia y metas educativas: Variables protectoras del rendimiento académico de bachilleres. Revista Colombiana de Psicología, 22(3), 241-252.

Gaxiola, J.C., Millanes, R.M. y Aranda, C.L. (2017). Estilos de crianza con experiencias de violencia de pareja. PSICUMEX, 7(2), 22-39.
González, S. y Rivera, M.A. (2016). Efectos del apoyo social, resiliencia y competencia social en el bienestar psicológico de adolescentes de sectores vulnerables, con eventos de vida estresantes. En J.C. Gaxiola y J. Palomar (Coords.), El bienestar psicológico: Una mirada desde Latinoamérica (pp. 31-60). Hermosillo, México: Qartuppi, S. de R.L. de C.V. Hirsch, A. (2012). Conductas no éticas en el ámbito universitario. Perfiles Educativos, XXXIV (Número Especial), 142-152.

Hirsch, A. (2016). Comportamiento responsable en la investigación y conductas no éticas en universidades de México y España. Revista de la Educación Superior, 46(179), 79-93.

Koepsell, D.R. y Ruiz de Chávez, M.H. (2015). Ética de la investigación: Integridad científica. México: CONBIOÉTICA/ CONACYT.

Piña, J.A. (en revisión). Sobre-explotación de la muestra en la investigación psicológica: ¿Es probable que dé lugar a conductas éticamente inaceptables y éticamente cuestionables?

Piña, J.A. (2015). Un análisis crítico del concepto de resiliencia en psicología. Anales de Psicología, 31(3), 751-758.

Piña, J.A. (2016). Errores conceptuales y metodológicos en psicología y salud: Estudio de caso sobre el uso y abuso del modelamiento con ecuaciones estructurales. Papeles del Psicólogo, 37(3), 231-239.

Piña, J.A. (2017). Predictores del rendimiento académico en bachilleres, de Gaxiola y colaboradores: ¿Un ejemplo de corrupción académica? Enseñanza e Investigación en Psicología, 22(2), 237-256.

Piña, J.A. (2018). Psicología y ética de investigación: 'Se deben usar instrumentos de medida sin probar sus propiedades psicométricas. Enseñanza e Investigación en Psicología, 23(1), 88-107.

Real Academia Española (2014). Diccionario de la Real Academia de la Lengua. Madrid: Espasa-Calpe.

Sociedad Mexicana de Psicología (2007). Código de ética del psicólogo. México: Trillas. 
Tabla 1.

Auto-plagios de Gaxiola y Frías, publicados en 2010 en dos capítulos: "Gaxiola y Frías (2010a): Usado por el primero en su reingreso al SNI en 2011, como Producción de Capítulos en Libros (Registro No. 2)"1 y "Gaxiola y Frías (2010b): Usado por el primero en su reingreso al SNI en 2011, como Producción de Artículos (Registro No. 4)"

Apartado

Introducción

Objetivo
Gaxiola y Frías (2010A): Usado por el Primero en SU REINGReso aL SNI eN 2011, COMO Producción de Capítulos en Libros (Registro No. 2)

(P. 75): Una posible definición del maltrato infantil es la propuesta por la Organización Mundial de la Salud que define el maltrato infantil como "Cualquier forma de daño físico y/o emocional, abuso sexual, negligencia o cualquier forma de trato negligente, comercial u explotación, que resulta en daño actual o potencial a la salud, sobrevivencia o desarrollo de la dignidad, en el contexto de una relación de responsabilidad, confianza o poder" (Organización Mundial de la Salud OMS/WHO, 1999, pp. 15-16).

(P. 76): El Sistema Nacional para el Desarrollo Integral de la Familia (DIF, 2004) registró para todo México de 1995 a 2000, un promedio de 25,000 casos de maltrato a menores cada año.

(P. 77): La calidad de vida es definida por el grupo de calidad de vida de la Organización Mundial de la Salud como "La percepción individual de la posición en la vida en el contexto de la cultura y el sistema de valores en el cual vive, y en relación con sus metas, expectativas, estándares y preocupaciones". (The WHOQoL Group, 1994, p. 43).

(P. 78): La teoría de los sistemas de desarrollo postula que la interacción entre los aspectos biológicos, psicológicos y sociales del ser humano afectan el curso del desarrollo de modos diferentes [...] estos pueden producir modos diferenciales de respuesta que culminan con patrones adaptativos o desadaptativos de comportamientos [...]

(P. 80): Atendiendo a lo señalado anteriormente, el objetivo de la presente investigación fue medir la relación entre el apoyo social y la autoestima en la calidad de vida de las mujeres con historia de maltrato infantil.
Gaxiola y Frías (2010b): Usado por el PRIMERO EN SU REINGRESO AL SNI EN 2011, COMO Producción de Artículos (Registro No. 4)

(P. 791): Una posible definición del maltrato infantil es la propuesta por la Organización Mundial de la Salud que define el maltrato infantil como “Cualquier forma de daño físico y/o emocional, abuso sexual, negligencia o cualquier forma de trato negligente, comercial u explotación, que resulta en daño actual o potencial a la salud, sobrevivencia o desarrollo de la dignidad, en el contexto de una relación de responsabilidad, confianza o poder" (Organización Mundial de la Salud OMS/WHO, 1999, pp. 15-16).

(P. 76): El Sistema Nacional para el Desarrollo Integral de la Familia (DIF, 2004) registró para todo México de 1995 a 2000, un promedio de 25,000 casos de maltrato a menores cada año.

(P. 791): La calidad de vida es definida por el grupo de calidad de vida de la Organización Mundial de la Salud como "La percepción individual de la posición en la vida en el contexto de la cultura y el sistema de valores en el cual vive, y en relación con sus metas, expectativas, estándares y preocupaciones". (The WHOQoL Group, 1994, p. 43).

(P. 791): La teoría de los sistemas de desarrollo postula que la interacción entre los aspectos biológicos, psicológicos y sociales del ser humano afectan el curso del desarrollo de modos diferentes [...] estos pueden producir modos diferenciales de respuesta que culminan con patrones adaptativos o desadaptativos de comportamientos [...].

(P.792): Atendiendo a lo señalado anteriormente, el objetivo de la presente investigación fue medir la relación entre el apoyo social y la autoestima en la calidad de vida de las mujeres con historia de maltrato infantil.

1 Dicha información fue proporcionada por el Sistema Nacional de Investigadores, a solicitud expresa del autor de este trabajo, a través del órgano de Transparencia y Acceso a la Información. 


\begin{tabular}{|c|c|c|}
\hline APARTADO & $\begin{array}{l}\text { Gaxiola y Frías (2010a): Usado por el PRimero en } \\
\text { Su reingreso al SNI en } 2011 \text {, como Producción } \\
\text { De Capítulos en Libros (Registro No. 2) }\end{array}$ & $\begin{array}{l}\text { Gaxiola y Frías (2010B): Usado por el } \\
\text { PRimero en su reingreso al SNI en } 2011 \text {, como } \\
\text { Producción de Artículos (Registro No. 4) }\end{array}$ \\
\hline
\end{tabular}

(Pp. 81-82): Se conformó un inventario para ser aplicado a las madres, que incluyó preguntas demográficas como la edad de la madre y su estatus matrimonial, el ingreso familiar mensual, el ingreso por separado para cada uno de los miembros de la pareja, la ocupación del compañero y el nivel educativo de los padres. Además, se aplicaron siete preguntas estructuradas de las Escalas de Tácticas de Conflicto de Strauss [...]

Las características de apoyo de la pareja fueron evaluadas con una serie de preguntas elaboradas especialmente para el estudio [...]

\section{Instrumentos}

La autoestima fue medida con 13 preguntas del inventario de Harterard, compilado en el proyecto DICA-R-7.3 [...]

El apoyo social se midió mediante un cuestionario de 18 preguntas conformado por dos escalas incluidas en el proyecto DICA-R-7.3 [...]

Por último, la calidad de vida fue medida con la versión breve del Inventario de Calidad de Vida [...]

P. 793): Se conformó un inventario para ser aplicado a las madres, además, se aplicaron siete preguntas de las Escalas de Tácticas de Conflictos de Strauss [...] para medir la historia de abuso.

Las características de apoyo de la pareja fueron evaluadas con una serie de preguntas con escala Likert elaboradas especialmente para el estudio [...]

La autoestima fue medida con 13 preguntas y el apoyo social mediante un cuestionario de 18, ambos el inventario de Harterard, compilado en el proyecto DICA-R-7.3 [...]

Por último, la calidad de vida fue medida con la versión breve del Inventario de Calidad de Vida [...]

Todas las alfas fueron superiores a .60 , por lo cual fueron consideradas aceptables en términos estadísticos [...]

Todas las alfas fueron superiores a .60 , por lo cual fueron consideradas aceptables en términos estadísticos [...]

(Pp. 82-83): Primeramente se obtuvieron las estadísticas univariadas, medias y desviaciones estándar para las variables continuas y frecuencias para las variables categóricas.

Análisis estadístico

Resultados
Se obtuvieron las alfas de todas las escalas y se corrió una matriz de correlaciones entre las variables protectoras asociadas a la calidad de vida, para posteriormente realizar un modelo de regresión a partir de las correlaciones significativas.

(P. 85): La tabla 4 resume el análisis de regresión lineal de las variables predictoras de la calidad de vida. Se encontró que las variables relación de pareja (Beta $.22, \mathrm{R} 2=.08$ ) y autoestima (Beta $.33, \mathrm{R} 2=.23$ ) predijeron de manera significativa $(\mathrm{p}<.0001)$ a la calidad de vida. Esto significa que entre mejores sean las relaciones de pareja y mayor su autoestima, las madres de la muestra tienen una mayor calidad de vida. El modelo obtuvo una varianza explicada de .25 lo que implica que una cuarta parte de la calidad de vida de las mujeres de la muestra es atribuible a las relaciones de pareja y a la autoestima.
(Pp. 793-894): Para el análisis de datos, primeramente se obtuvieron las estadísticas univariadas, medias y desviaciones estándar para las variables continuas y frecuencias para las variables categóricas.

Se obtuvieron las alfas de todas las escalas y se corrió una matriz de correlaciones entre las variables protectoras asociadas a la calidad de vida, para posteriormente realizar un modelo de regresión a partir de las correlaciones significativas.

(P. 794): La tabla 2 resume el análisis de regresión lineal de las variables predictoras de la calidad de vida. Se encontró que las variables relación de pareja (Beta $.22, \mathrm{R} 2=.08$ ) y autoestima (Beta $.33, \mathrm{R} 2=.23$ ) predijeron de manera significativa $(p<.0001)$ a la calidad de vida. Esto significa que entre mejores sean las relaciones de pareja y mayor su autoestima, las madres de la muestra tienen una mayor calidad de vida. El modelo obtuvo una varianza explicada de .25 lo que implica que una cuarta parte de la calidad de vida de las mujeres de la muestra es atribuible a las relaciones de pareja y a la autoestima. 


\begin{tabular}{|c|c|}
\hline APARTADO & 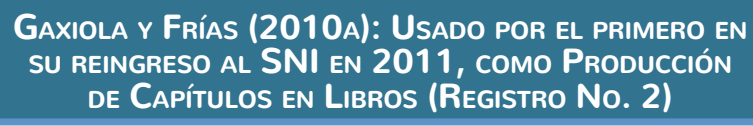 \\
\hline \multirow[b]{3}{*}{ Discusión } & $\begin{array}{l}\text { (P. 85): De acuerdo con la teoría de los sistemas } \\
\text { de desarrollo algunas personas en situaciones de } \\
\text { riesgo podrían tener calidad de vida a partir de } \\
\text { la interacción con variables protectoras [...] En la } \\
\text { presente investigación las variables predictoras } \\
\text { de la calidad de vida en la muestra fueron la } \\
\text { autoestima y la relación de pareja. }\end{array}$ \\
\hline & $\begin{array}{l}\text { La autoestima puede constituirse en una variable } \\
\text { protectora al mejorar la salud, el comportamiento } \\
\text { social positivo y la calidad de vida [...] Los datos } \\
\text { avalan la importancia de la autoestima en la calidad } \\
\text { de vida, aunque existe una discusión que vincula } \\
\text { a la autoeficacia, es decir la autoestima puesta } \\
\text { en acción, con mayores beneficios como variable } \\
\text { protectora [...], lo cual debe estudiarse en futuras } \\
\text { investigaciones. }\end{array}$ \\
\hline & $\begin{array}{l}\text { (P. 86): DeLongis et al [...] encontraron que el apoyo } \\
\text { brindado entre las parejas influye en su afecta } \\
\text { positivo y esto a su vez afecta el bienestar de éstas. } \\
\text { Por su parte, Purdom, Lucas y Miller [...] señalaron } \\
\text { que al incrementarse el apoyo del esposo, la } \\
\text { satisfacción de la pareja se incrementa. A partir de } \\
\text { estos hallazgos el fomentar las relaciones de apoyo } \\
\text { de los hombres hacia las mujeres víctimas del } \\
\text { maltrato infantil puede mejorar finalmente la calidad } \\
\text { de vida de esta población. }\end{array}$ \\
\hline
\end{tabular}

(P. 86): A partir de los datos revisados en el presente estudio, se muestra que la calidad de vida como índice del estado de satisfacción en diversas áreas de la vida constituye el resultado de diversas interacciones con los contextos que rodean a las personas, incluso en poblaciones viviendo en situaciones de riesgo. En futuras investigaciones es necesaria la medición de otras variables que puedan afectar la calidad de vida de estas poblaciones, con el fin de complementar los datos del presente estudio.
Gaxiola y Frías (2010B): Usado poR el PRIMERO EN SU REINGRESO AL SNI EN 2011, COMO Producción de Artículos (Registro No. 4)

(P. 85): De acuerdo con la teoría de los sistemas de desarrollo algunas personas en situaciones de riesgo podrían tener calidad de vida a partir de la interacción con variables protectoras [...] En la presente investigación las variables predictoras de la calidad de vida en la muestra fueron la autoestima y la relación de pareja.

La autoestima puede constituirse en una variable protectora al mejorar la salud, el comportamiento social positivo y la calidad de vida [...] Los datos avalan la importancia de la autoestima en la calidad de vida, aunque existe una discusión que vincula a la autoeficacia, es decir la autoestima puesta en acción, con mayores beneficios como variable protectora [...], lo cual debe estudiarse en futuras investigaciones.

(P. 794): DeLongis et al [...] encontraron que el apoyo brindado entre las parejas influye en su afecta positivo y esto a su vez afecta el bienestar de éstas. Por su parte, Purdom, Lucas y Miller [...] señalaron que al incrementarse el apoyo del esposo, la satisfacción de la pareja se incrementa. A partir de estos hallazgos el fomentar las relaciones de apoyo de los hombres hacia las mujeres víctimas del maltrato infantil puede mejorar finalmente la calidad de vida de esta población.

(P. 795): A partir de los datos revisados en el presente estudio, se muestra que la calidad de vida como índice del estado de satisfacción en diversas áreas de la vida constituye el resultado de diversas interacciones con los contextos que rodean a las personas, incluso en poblaciones viviendo en situaciones de riesgo. En futuras investigaciones es necesaria la medición de otras variables que puedan afectar la calidad de vida de estas poblaciones, con el fin de complementar los datos del presente estudio. 
Tabla 2.

Datos estadísticos en dos reportes de investigación publicados por Gaxiola y colaboradores en 2012 y 2013.

$\begin{array}{ccc}\text { Reporte } 1 \text { publicado en la Revista de } & \text { Reporte } 3 \text { publicado en la Revista } \\ \text { Indicadores } & \text { Psicología de la PUCP (Gaxiola, González, } & \text { Interamericana de Psicología (Gaxiola, } \\ & \text { Contreras y Gaxiola, 2012). } & \text { González, Domínguez y Gaxiola, 2013). }\end{array}$

Grupo no resiliente:

$x^{2}=80.1$

GI. $=15$

$P=.64$

$\mathrm{BBNFI}=.96$

$B B N N F I=1.0$

$\mathrm{CFI}=1.0$

RMSEA $=.00$

$R^{2}=.00$ (lo que significa que no hay varianza explicada para el rendimiento académico)

Grupo resiliente:

$x^{2}=47.2$

$\mathrm{Gl} .=15$

$P=.410$

$\mathrm{BBNFI}=.84$

$\mathrm{BBNNFI}=.98$

$\mathrm{CFI}=.99$

RMSEA $=.02$

$R^{2}=.13$ (lo que significa que la varianza

explicada para el rendimiento académico es de $13 \%)$

Grupo no resiliente:

Amigos de riesgo con Vecindario de riesgo: .33 Amigos de riesgo con Autor-regulación: -.40 Auto-regulación con Resiliencia: .32

Resiliencia con Metas educativas: .55

Vecindario de riesgo con Metas educativas: -.38

Pesos factoriales
Grupo no resiliente:

$x^{2}=80.1$

$\mathrm{GI} .=15$

$P=.64$

$\mathrm{BBNFI}=.96$

$\mathrm{BBNNFI}=1.0$

$\mathrm{CFI}=1.0$

RMSEA $=.00$

$R^{2}=.00$ (lo que significa que no hay varianza explicada para el rendimiento académico)

Grupo resiliente:

$x^{2}=47.2$

$\mathrm{Gl} .=15$

$P=.410$

$\mathrm{BBNFI}=.84$

$\mathrm{BBNNFI}=.98$

$\mathrm{CFI}=.99$

RMSEA $=.02$

$R^{2}=.13$ (lo que significa que la varianza explicada para el rendimiento académico es de $13 \%)$

Grupo no resiliente:

Vecindario de riesgo con Amigos de riesgo: .33 Amigos de riesgo con Autor-regulación: - .40 Auto-regulación con Resiliencia: .35

Resiliencia con Metas educativas: .55

Vecindario de riesgo con Metas educativas: -.39

Grupo resiliente:

Vecindario de riesgo con Auto-regulación: -.25

Auto-regulación con resiliencia: .50

Resiliencia con Metas educativas: .43

Metas educativas con Rendimiento: .27 
Tabla 3.

Reportes de investigación de Gaxiola y colaboradores, por tipos de muestras y por objetivo.

\section{Publicación y N de LA muestra}

Objetivo

(S/P): Con base en lo anterior, el objetivo de la esta investigación fue identificar las 102 estudiantes de bachillerato. Nota: $54 \%$ eran hombres y $40 \%$ mujeres, con una edad promedio de 16 años (D.E $=1.12$ ). El $46.1 \%$ perteneciente al primer semestre, el $33.3 \%$ de tercero y el $20.6 \%$ de quinto semestre.

2. Gaxiola, González y Contreras (2012): 96 estudiantes de bachillerato.

Nota: $57.3 \%$ eran hombres y $42.7 \%$ mujeres, con una edad promedio de 16 años (D.E. = 1.12). El 46.1\% perteneciente al primer semestre, el $33.3 \%$ de tercero y el $20.6 \%$ de quinto semestre.

3. Gaxiola, González, Contreras y Gaxiola (2012): 120 estudiantes de bachillerato.

4. Gaxiola, González, Domínguez y Gaxiola (2013): 120 estudiantes de bachillerato.

5. Gaxiola y Frías (2008): 182 madres de familia.
(Pp. 168-169): Con base en lo anterior, esta investigación buscó medir de manera exploratoria las interrelaciones entre las variables internas: metas y resiliencia; y las variables externas: las características del barrio y las características de los amigos, y su posible predicción del rendimiento académico de estudiantes de preparatoria.

(P. 54): A partir de la revisión anterior, el objetivo de la presente investigación fue probar dos modelos estructurales de variables contextuales e individuales del grupo resiliente y del grupo no resiliente, y sus interrelaciones con el rendimiento escolar [...]

(Pp. 72-73): A partir de la revisión anterior, el objetivo de la presente investigación fue probar dos modelos estructurales de variables contextuales e individuales del grupo de resiliencia y no resiliencia, y sus interrelaciones con el rendimiento escolar [...]

(P. 20): Considerando lo anterior, el objetivo planteado para la presente investigación fue el identificar los factores que protegen a las madres de los factores de riesgo como son el vecindario inseguro, la violencia de pareja, la depresión, las creencias disciplinarias con relación al castigo y la historia de abuso, en la transmisión de la violencia manifestada como abuso infantil.
Características

Nótese que a pesar de que las muestras son "distintas", el objetivo en las publicaciones numeradas con 1 y 2 es el mismo.

Además, extraño y digno de mención es que, si las características sociodemográficas de edad y los porcentajes de partencia a los semestres eran los mismos, ¿cómo explicar ello si en el numerado con 1 la muestra era de $\mathrm{N}$ = 102 estudiantes y en el 2 de $\mathrm{N}=96$ estudiantes? ¿Cómo explicar también que los porcentajes de hombres y mujeres fueron diferentes?

Nótese que las muestras son las mismas y el objetivo en las publicaciones numeradas con 3 y 4 es el mismo. Ambas publicaciones fueron analizadas en el trabajo de Piña (2017). Sólo se modificó grupo resiliente y no-resiliente por grupo de resiliencia y no-resiliencia.

Nótese que en términos generales el objetivo descrito en las publicaciones numeradas con 5 y 6 es prácticamente el mismo, sólo que en uno (el 5) se incluyeron más factores para los análisis. 
Publicación y N de la muestra

6. Gaxiola, Frías y Figueredo (2011): 182 madres de familia.

7. Gaxiola, Gaxiola, González y Valenzuela (2014): 254 madres de familia

8. Gaxiola (2016): 254 madres de familia

9. Gaxiola y Gaxiola (2016): 254 madres de familia

10. Gaxiola, Gaxiola y Frías (2017): 254 madres de familia

11. Gaxiola, Millanes y Aranda (2017): 254 madres de familia

\section{Objetivo}

\section{Características}

(P. 32): Por tal motivo, el objetivo de la presente investigación fue identificar los factores que protegen a las madres, frente a la probabilidad de utilizar con sus hijos los estilos de crianza autoritarios, ante la presencia de riesgos específicos como la inseguridad del vecindario, la violencia de pareja y la depresión.

(P. 557): A partir de lo anterior, el objetivo del presente estudio fue evaluar las relaciones directas e indirectas, entre las variables contextuales apoyo social familiar, cohesión social del vecindario, y las variables individuales salud percibida, autorregulación, autoestima y bienestar psicológico, en sus efectos sobre la crianza positiva materna.

(P. 48): A partir de la revisión anterior se planteó el objetivo de probar los efectos directos e indirectos de los diversos tipos de apoyo social agrupados en un factor, la depresión y la participación de los problemas de autorregulación, el bienestar psicológico y la crianza autoritativa en el contexto de violencia de pareja.

(P. 144): A partir de lo anterior, el objetivo de la presente investigación fue probar un modelo de ecuaciones estructurales de los efectos del apoyo social percibido por las madres víctimas de violencia de pareja en las disposiciones a la resiliencia, el bienestar psicológico y la crianza autoritativa.

(P. 3): A partir de lo anterior, el objetivo del presente estudio fue medir las relaciones directas e indirectas, entre las variables contextuales apoyo social familiar, cohesión social del vecindario, y las variables individuales salud percibida, autorregulación, autoestima y bienestar psicológico, en sus efectos sobre la crianza positiva materna.

(P. 26): El objetivo del presente estudio fue evaluar los factores de riesgo de la violencia de pareja: depresión, problemas de autorregulación y maltrato infantil, así como los factores protectores: el apoyo social percibido de los amigos, familiares y vecinos, bienestar psicológico, disposiciones a la resiliencia, que hacen probable la crianza autoritativa.
Nótese que en las publicaciones numeradas con $7,8,9,10$ y 11 los objetivos son prácticamente los mismos, sólo que unas unas fueron analizando las relaciones entre ciertos factores; esto es, se fueron añadiendo o eliminando a conveniencia algunos factores. Por ejemplo, en una se analizó el factor salud percibida (números 7 y 10), mientras que en otras se eliminó (números 8 y 9). 
Tabla 5.

Ejemplos de alteración o vicio en el desarrollo de los textos, al comparar la Tesis Doctoral de Gaxiola y del libro publicado en 2014 (en coautoría con Frías y Corral). ${ }^{2}$

\begin{tabular}{|c|c|c|c|}
\hline APARTADO & $\begin{array}{l}\text { TESIS DOCTORAL }(2007) \\
\text { DEFENDIDA POR GAXIOLA Y } \\
\text { DIRIGIDA POR FrÍAS }\end{array}$ & $\begin{array}{l}\text { LiBro }(2014) \text { con AUTORÍA de } \\
\text { GaxiOLA, Frías y CorRAL }\end{array}$ & Comentarios \\
\hline
\end{tabular}

(P. 12): [...]: El maltrato infantil o abuso infantil es considerado un problema de salud mundial, debido a

Introducción

\section{Capítulo 1} su distribución en todos los países y a las repercusiones que provoca en la integridad física o psicológica en millones de niños (OMS, 1997).

\section{(P. 19): El maltrato} infantil implica enfrentar circunstancias ambientales adversas en la vida de los niños que afectan el curso del desarrollo normalmente organizado y adaptativo y lo transforman en un curso menos predictivo y adaptativo, debido a que dichas circunstancias constituyen eventos incontrolables (Wolfe y Yuan, 2001).

(P. 21): Algunos investigadores sitúan al castigo corporal $y$ al maltrato infantil como fenómenos situados en el mismo continuo de acciones dirigidas hacia los niños, de tal manera que si las diferencias son de grado, el maltrato físico ocupa los extremos del continuo donde pueden situarse acciones como el provocar heridas, fracturas o quemaduras a los niños (Strauss, 1994; OMS, 2002).
(P. 13): [...]: El maltrato infantil o abuso infantil es considerado un problema de salud mundial, debido a su distribución en todos los países y a las repercusiones que provoca en la integridad física o psicológica en millones de niños (Secretaría de Salud, 2006).

\section{(P. 21): El maltrato} infantil implica enfrentar circunstancias ambientales adversas en la vida de los niños que afectan el curso del desarrollo normalmente organizado y adaptativo y lo transforman en un curso menos predictivo y adaptativo, debido a que dichas circunstancias constituyen eventos incontrolables (Wolfe y Yuan, 2001; Widom, 2014).

(P. 24): Algunos investigadores identifican al castigo corporal $y$ al maltrato infantil como fenómenos situados en el mismo continuo de acciones dirigidas hacia los niños, de tal manera que si las diferencias son de grado, el maltrato físico ocupa los extremos del continuo donde pueden situarse acciones como el provocar heridas, fracturas o quemaduras a los niños (Strauss, 1994; OMS, 2002; Widom, 2014).
Nótese el cambio de referencia.
Nótese el cambio de una palabra por otra, el añadido de una referencia o inclusive el añadido de un factor de análisis distinto.

2 Los resaltados con negritas son míos. Nótese que en ambos productos los párrafos son iguales, que se trató de la misma muestra y que los resultados son también los mismos 
Tesis Doctoral (2007) DEFENDIDA POR GAXIOLA Y DIRIGIDA POR FríAS

(P. 21): El abuso emocional incluye críticas repetidas, denigrar o aterrorizar al niño, aislarlo de otros o rechazarlo explícitamente (Feiring, 2005; OMS, 2002). El daño emocional, de acuerdo con Sneddo (2003), puede ser causado por la hostilidad verbal, las expectativas irreales, el miedo y la ansiedad inducida, las amenazas de abandono, el rechazo, la deprivación de la atención y estimulación o por ejercer poder excesivo sobre el menor.

(P. 22): [...] El maltrato infantil tiene también repercusiones a largo plazo en la salud física de quienes lo padecen, por ejemplo síndromes de dolor crónico (Heim et al., 1998), síndrome de fatiga crónica (Houdenhove et al., 2001) y colon irritable (Leserman et al. 1997).

(P. 23): El abuso infantil no solamente afecta la salud física y la seguridad de los niños, además puede afectar la visión que ellos tienen del mundo, las relaciones sociales, el ajuste psicológico de aquellos que lo experimentan (Cicchetti y Toth, 2000; Emery y LaumannBillings, 1998).
LiBro (2014) CON AUTORÍA DE Gaxiola, Frías y Corral

Comentarios

(P. 25): El abuso emocional incluye críticas repetidas, denigrar o aterrorizar al niño, aislarlo de otros o rechazarlo explícitamente (Feiring, 2005; OMS, 2002). El daño emocional puede ser causado por la hostilidad verbal, las expectativas irreales, el miedo y la ansiedad inducida, las amenazas de abandono, el rechazo, la deprivación de la atención y estimulación o por ejercer poder excesivo sobre el menor (Sneddo, 2003; Tommyr, Draca, Cain y MacMillan, 2011).

(P. 25): [...] El maltrato infantil tiene también repercusiones a largo plazo en la salud física de quienes lo padecen, por ejemplo síndromes de dolor crónico (Heim, Herlerth, Hanker y Hellhammer, 1998), síndrome de fatiga crónica (Houdenhove, Neerinckx y Lyens, 2001), colon irritable (Leserman, Zhiming, Dross,am, Toomey, Nachman y Glogau, 1997), y desnutrición (Widom, Czaja, Bentley y Johnson, 2012).

(P. 27): El abuso infantil no solamente afecta la salud física y la seguridad de los niños, además puede afectar la visión que ellos tienen del mundo, las relaciones sociales, el ajuste psicológico de aquellos que lo experimentan (Cicchetti y Toth, 2000; Emery y LaumannBillings, 1998; Widom, 2014). 
Apartado

Participantes

\begin{tabular}{|c|c|c|}
\hline & $\begin{array}{l}\text { (P. 87): Tabla 1. } \\
\text { demográficas de }\end{array}$ & $\begin{array}{l}\text { riables } \\
\text { muestra: }\end{array}$ \\
\hline & $\begin{array}{l}\text { Variable } \\
\text { D.E. }\end{array}$ & $\mathrm{M}$ \\
\hline & Edad & $\begin{array}{l}(\mathrm{N}=182) \\
34.8\end{array}$ \\
\hline $\begin{array}{l}\text { Resultados: Variables } \\
\text { demográficas }\end{array}$ & $\begin{array}{l}6.3 \\
\text { Tiempo de vivir } \\
5.1\end{array}$ & 12.4 \\
\hline & $\begin{array}{l}\text { con pareja } \\
\text { Ingreso de pareja } \\
7405.8\end{array}$ & 6058.3 \\
\hline & $\begin{array}{l}\text { Ingreso propio } \\
3539.1 \\
{[\ldots]}\end{array}$ & 1609.3 \\
\hline
\end{tabular}

\section{Tesis Doctoral (2007) DEFENDIDA POR GAXIOLA Y DIRIGIDA POR Frías \\ LIBRO (2014) CON AUTORÍA DE Gaxiola, Frías y Corral}

\section{Comentarios}

(Pp. 80-81): Se entrevistaron

182 madres de familia en situaciones de riesgo para conformar un muestreo deliberado por heterogeneidad (Cook y Campbell, 1979) que cumplieron con los siguientes requisitos:

A. Con relación de pareja estable durante el último año. $B$. Con al menos un hijo entre 6 y 12 años.

Las variables de riesgo que fueron tomados en cuenta para la conformación de la muestra fueron:

a. Madres que acudían en buscar atención médica en los hospitales tanto públicos como privados de la ciudad de Hermosillo, Sonora.

b. Madres con niños en escuelas de educación especial públicas y privadas de la ciudad de Hermosillo, Sonora. c. Madres con número de hijos arriba del promedio para el Estado. De acuerdo con los datos del INEGI del Censo de Población y Vivienda del año 2000, en el Estado de Sonora existe un promedio de 2.6 por familia (INEGI, 2001), por lo cual, puede ser un factor de riesgo en la ciudad de Hermosillo, Sonora el tener 4 hijos o más por familia [...]

\section{(P. 87): Tabla 1. Variables}

(P. 107): Tabla 1. Variables demográficas de la muestra:

Variable

D.E.

Edad

6.3

Tiempo de vivir

5.1

con pareja

Ingreso de pareja $\quad 6058.3$

7405.8

Ingreso propio $\quad 1609.3$

3539.1

[...]
Las variables de riesgo que para la conformación de la a. Madres que acudían en atención médica en ados de la ciudad de

de educación espe termosillo, Sonora. arriba del promedio para el or familia (INEGI, 2010), por hijos o más por familia [...] Población y Vivienda del año
M

$(\mathrm{N}=182)$ 34.8

12.4
Nótese el cambio en el dato del promedio de número de hijos y en la fecha de la referencia del INEGI. 


\begin{tabular}{|c|c|c|c|}
\hline ApARTADo & $\begin{array}{l}\text { TeSIS DOCTORAL (2007) } \\
\text { DEFENDIDA POR GAXIOLA Y } \\
\text { DIRIGIDA POR FRÍAS }\end{array}$ & $\begin{array}{l}\text { LiBro }(2014) \text { con aUtoría de } \\
\text { GaXiola, Frías y Corral }\end{array}$ & CoMENTARIOS \\
\hline
\end{tabular}

Resultados: Alfas de los instrumentos
(Pp. 88-89): Tabla 3. Alfas de las Escalas empleadas en la Investigación.

(Pp. 108): Tabla 3. Alfas de las escalas empleadas en la investigación.

\begin{tabular}{|c|c|}
\hline $\begin{array}{ll}\text { Escala } & \text { Alfa } \\
\text { de Cronbach } & \end{array}$ & $\begin{array}{l}\text { Escala } \\
\text { de Cronbach }\end{array}$ \\
\hline Abuso infantil & Abuso infantil \\
\hline 0.75 & 0.75 \\
\hline Historia de abuso & Historia de abuso \\
\hline 0.89 & 0.89 \\
\hline Apoyo social (exosistema...) & Apoyo social (exosistema...) \\
\hline 0.90 & 0.90 \\
\hline Apoyo social (microsistema...) & Apoyo social (microsistema...) \\
\hline 0.79 & 0.79 \\
\hline Cohesión del vecindario & Cohesión del vecindario \\
\hline 0.85 & 0.85 \\
\hline Apoyo de pareja & Apoyo de pareja \\
\hline 0.93 & 0.93 \\
\hline Violencia de pareja & Violencia de pareja \\
\hline 0.93 & 0.93 \\
\hline Vecindario peligroso & Vecindario peligroso \\
\hline 0.92 & 0.92 \\
\hline Autoestima & Autoestima \\
\hline 0.84 & 0.84 \\
\hline Adaptabilidad autoritaria & Adaptabilidad autoritaria \\
\hline 0.93 & 0.93 \\
\hline Estilo autoritario & Crianza autoritaria \\
\hline 0.78 & 0.78 \\
\hline Estilo permisivo & Crianza permisiva \\
\hline 0.94 & 0.94 \\
\hline Crianza & Crianza autoritaria \\
\hline 0.84 & 0.84 \\
\hline Creencias & Creencias \\
\hline 0.78 & 0.78 \\
\hline Factor $\mathrm{K}$ & Factor $\mathrm{K}$ \\
\hline 0.82 & 0.82 \\
\hline Depresión & Depresión \\
\hline 0.97 & 0.97 \\
\hline Calidad o & Calidad de vida \\
\hline 0.90 & 0.90 \\
\hline
\end{tabular}

Alfa

de Cronbach

0.75

0.89

0.90

Cohesión del vecindario

0.93

Autoestima

Adaptabilidad autoritaria

0.78

0.94

0.78

0.82

0.97

0.90 
APARTADO

(a)

Resultados: Modelamiento con ecuaciones estructurales

Resultados: Modelamiento con ecuaciones estructurales

\section{Tesis Doctoral (2007) DEFENDIDA POR GAXIOLA Y DIRIGIDA POR Frías}

(Pp. 91-92): Las variables que conforman a los factores protectores (ver Figura 4) son el exosistema (lambda=.39), el microsistema (.79) y el ontosistema protector (.59). El exosistema protector estuvo conformado con las variables apoyo social y apoyo del vecindario; el microsistema protector con las variables relación de pareja y apoyo social de familiares y amigos; mientras el ontosistema protector lo formaron las variables autoestima y calidad de vida. Los factores de riesgo constituidos por los siguientes sistemas de riesgo: el exosistema, conformado por el vecindario inseguro (lambda=.23), el microsistema conformado por la violencia de pareja (.76) y el ontosistema de riesgo (.63). El ontosistema de riesgo estuvo conformado por la depresión, la historia de abuso y las creencias disciplinarias con respecto al castigo. Los indicadores del ontosistema y la variables estilos de crianza fueron obtenidos considerando las correlaciones de Pearson $(p<.0001)$ entre sus constituyentes (ver Tabla 4).

\section{(P. 93): Nota al pie de la} Figura 4. Modelo estructural. Factores protectores y de riesgo con sus efectos sobre la adaptabilidad de los estilos de crianza y el abuso infantil.

$$
\begin{aligned}
& X 2=68.6 \\
& 24 \mathrm{G} . \text { de } \mathrm{L} . \\
& \mathrm{p}=0.00000 \\
& \mathrm{BBNFI}=.85 \\
& \mathrm{BBNNFI}=.87 \\
& \mathrm{CFI}=.91 \\
& \mathrm{RMSEA}=.07 \\
& \mathrm{R} 2=.13
\end{aligned}
$$

\section{LiBro (2014) CON AUTORÍA DE} Gaxiola, Frías y Corral

(P. 111): Las variables que conforman a los factores protectores (ver figura 4) son el exosistema (lambda= .39), el microsistema (.79) y el ontosistema protector (.59). EI exosistema protector estuvo conformado con las variables apoyo social y apoyo del vecindario; el microsistema protector con las variables relación de pareja y apoyo social de familiares y amigos; mientras el ontosistema protector lo formaron las variables autoestima y calidad de vida. Los factores de riesgo constituidos por los siguientes sistemas de riesgo: el exosistema, conformado por el vecindario inseguro (lambda $=.23)$, el microsistema conformado por la violencia de pareja (.76) y el ontosistema de riesgo (.63). El ontosistema de riesgo estuvo conformado por la depresión, la historia de abuso y las creencias disciplinarias con respecto al castigo. Los indicadores del ontosistema y la variables estilos de crianza fueron obtenidos considerando las correlaciones de Pearson $(p<.0001)$ entre sus constituyentes (ver Tabla 4).

(P. 112): Nota al pie de la Figura 4. Modelo estructural. Factores protectores y de riesgo con sus efectos sobre la adaptabilidad de los estilos de crianza y el abuso infantil.

$$
\begin{aligned}
& X 2=68.6 \\
& 24 \mathrm{G} . \text { de } \mathrm{L} . \\
& \mathrm{p}=0.00000 \\
& \mathrm{BBNFI}=.85 \\
& \mathrm{BBNNFI}=.87 \\
& \mathrm{CFI}=.91 \\
& \mathrm{RMSEA}=.07 \\
& \mathrm{R} 2=.13
\end{aligned}
$$




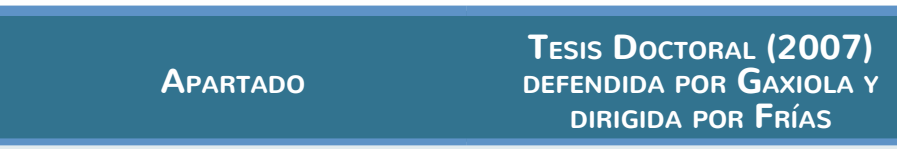

(Pp. 93-94): Los resultados de la investigación aquí reportada aportan datos sobre la influencia de la ecología de las variables protectoras en las variables de riesgo presentes en el entorno de las madres encuestadas y su influencia sobre la adaptabilidad de los estilos de crianza y reproducción del abuso infantil. Los datos muestran influencias directas entre los factores protectores, la adaptabilidad de los estilos de crianza y el abuso infantil, mediados por los factores de riesgo.

Al ponerse a prueba la teoría transaccional que considera que hay una interacción entre los factores protectores y de riesgo y que provocan respuestas positivas 0 negativas de acuerdo al peso específico de cada uno de ellos (Cicchetti y Lynch, 1993), los datos obtenidos indicaron que no se presenta interacción entre esos factores para las variables dependientes del abuso infantil o adaptabilidad de la crianza. De esta manera, la teoría transaccional por lo menos en esta muestra no fue comprobada, en lugar de ello los resultados del modelo estructural mostraron que la influencia entre los factores protectores y de riesgo es de carácter aditivo.

(P. 94): Los datos avalan los procesos de operación de los factores protectores según los diversos modelos mencionados por Werner (1993b) debido a que las variables protectoras estuvieron combinadas aditivamente para contrarrestar las variables de riesgo; los efectos de los factores protectores disminuyeron con la cantidad de riesgos y los factores protectores no tuvieron efectos significativas sin la presencia de los factores de riesgo.
LIBRo (2014) CON AUTORÍA DE

Gaxiola, Frías y Corral

Comentarios

(Pp. 113): Los resultados de la investigación aquí reportada aportan datos sobre la influencia de la ecología de las variables protectoras en las variables de riesgo presentes en el entorno de las madres encuestadas y su influencia sobre la adaptabilidad de los estilos de crianza y reproducción del abuso infantil. Los datos muestran influencias directas entre los factores protectores, la adaptabilidad de los estilos de crianza y el abuso infantil, mediados por los factores de riesgo.

Al ponerse a prueba la teoría transaccional que considera que hay una interacción entre los factores protectores $y$ de riesgo y que provocan respuestas positivas o negativas de acuerdo al peso específico de cada uno de ellos (Cicchetti y Lynch, 1993), los datos obtenidos indicaron que no se presenta interacción entre esos factores para las variables dependientes del abuso infantil o adaptabilidad de la crianza. De esta manera, la teoría transaccional por lo menos en esta muestra no fue comprobada, en lugar de ello los resultados del modelo estructural mostraron que la influencia entre los factores protectores y de riesgo es de carácter aditivo.

(P. 113): Los datos avalan los procesos de operación de los factores protectores según los diversos modelos mencionados por Werner (1993b) debido a que las variables protectoras estuvieron combinadas aditivamente para contrarrestar las variables de riesgo; los efectos de los factores protectores disminuyeron con la cantidad de riesgos y los factores protectores no tuvieron efectos significativas sin la presencia de los factores de riesgo. 
Tesis Doctoral (2007) DEFENDIDA POR GAXIOLA Y DIRIGIDA POR Frías

(P. 94): Los datos de la investigación también sustentan lo señalado por Kotliarenco et al. (1997) que sugieren que los factores protectores tienen sus efectos indirectos en la resiliencia a partir de su relación con la variable de riesgo., De esta manera, las variables protectoras manifiestan su influencia debido a la presencia de estresares en el entorno de las personas (Cicchetti y Rogosch, 1997; Muller y Lemieux, 2000; Rutter, 2007).

(P. 95): [...] De acuerdo con Sampson et al. (1997) los modelos de eficacia colectiva postulan que los adultos en los vecindarios socialmente cohesivos establecen contextos de apoyo para la socialización de los niños del vecindario. Los vecindarios pueden de este modo moderar las influencias de entornos más inmediatos como el ambiente familiar en el desarrollo de los niños (Boyce et al., 1998). Silk et al. (2004) reportaron que la cohesión social del vecindario es un factor protector del los estilos maternales hostiles.
LiBRo (2014) CON AUTORÍA DE Gaxiola, Frías y Corral

\section{Comentarios}

(P. 114): Los datos de la investigación también sustentan lo señalado por Kotliarenco, Cáceres y Fontecilla (1997) que sugieren que los factores protectores tienen sus efectos indirectos en la resiliencia a partir de su relación con la variable de riesgo. De esta manera, las variables protectoras manifiestan su influencia debido a la presencia de estresares en el entorno de las personas (Cicchetti y Rogosch, 1997; Muller y Lemieux, 2000; Rutter, 2007).

(Pp. 114-115): [...] De acuerdo con Sampson, Raudenbush y Earls (1997), los modelos de eficacia colectiva postulan que los adultos en los vecindarios socialmente cohesivos establecen contextos de apoyo para la socialización de los niños del vecindario. Los vecindarios pueden, de este modo, moderar las influencias de entornos más inmediatos como el ambiente familiar en el desarrollo de los niños (Boyce, Frank, Jensen y otros, 1998). Silk, Sessa, Morris y otros (2004) reportaron que la cohesión social del vecindario es un factor protector del los estilos maternales hostiles, lo cual es apoyado también por otros estudios (Byrnes y Miller, 2012; Meunier, Wade y Jenkins, 2012).
Nótese el añadido de una referencia actualizada. 
Tesis Doctoral (2007)

DEFENDIDA POR GAXIOLA Y DIRIGIDA POR FRÍAS
LiBro (2014) CON AUTORÍA DE Gaxiola, Frías y Corral
(P. 98): La historia de abuso fue otro indicador del ontosistema de riesgo. Las consecuencias de la historia de abuso como factor de riesgo a largo plazo han sido establecidas en investigaciones internacionales (Elam y Kleist, 1999; Carlson et al., 2003; Frías, 2002; Malinowsky-Rumell y Hansen, 1993; Wissow, 1995) y otras llevadas a cabo en la región (Frías, 2002; Frías y Gaxiola, 2005).

(P. 100): El presente estudio puede servir para justificar la exploración de otras variables que en la localidad funcionan como protectoras y que en otros países no funcionan como tales.

(P. 101): Entre las limitaciones del estudio se encuentra el hecho de que es una investigación retrospectiva que puede presentar olvidos de la información. También pudo verse afectada por las circunstancias presentes en la vida de las madres encuestadas o bien por el mismo contexto de la entrevista. Otra de las limitaciones es que se la información se obtuvo por medio del reporte verbal de las madres. Las investigaciones subsiguientes pueden utilizar registros observacionales de la adaptabilidad de los estilos de la crianza de las madres ante la presencia de los hijos bajo criterios de logro, atendiendo el concepto de competencias de Ribes (1990a).
(P. 119): La historia de abuso fue otro indicador del ontosistema de riesgo. La historia de abuso en el modelo resultante fue una variable de riesgo para la reproducción del abuso de las madres de esta muestra. Las consecuencias de la historia de abuso como factor de riesgo a largo plazo han sido establecidas en investigaciones internacionales (Carlson, McNutt y Choi, 2003; Frías, 2002; MalinowskyRumell y Hansen, 1993; Valentino, Nuttall, Comas, Borkowski y Akai, 2012) y otras llevadas a cabo en la región (Frías, 2002; Frías y Gaxiola, 2005).

(P. 121): El presente estudio puede servir para justificar la exploración de otras variables que en la localidad funcionan como protectoras y que en otros países no funcionan como tales.

(P. 122): Entre las limitaciones del estudio se encuentra el hecho de que es una investigación retrospectiva que puede presentar olvidos de la información. También pudo verse afectada por las circunstancias presentes en la vida de las madres encuestadas o bien por el mismo contexto de la entrevista. Otra de las limitaciones es que se la información se obtuvo por medio del reporte verbal de las madres. Las investigaciones subsiguientes pueden utilizar registros observacionales de la adaptabilidad de los estilos de la crianza de las madres ante la presencia de los hijos bajo criterios de logro, atendiendo el concepto de competencias de Ribes (1990a).
Nótese el añadido de un párrafo y de una referencia actualizada. 